\title{
Orbit Determination Using TDMA Radio Navigation Data with Implicit Measurement Times
}

\author{
Ryan C. Dougherty* and Mark L. Psiaki ${ }^{\dagger}$ \\ Cornell University, Ithaca, New York, 14853-7501
}

\begin{abstract}
An orbit determination Kalman filter has been developed that uses two types of measurements of time division multiple access (TDMA) L-Band signals from satellites in low Earth orbit, a carrier phase measurement and a biased pseudorange measurement. This algorithm will enable precise orbit determination of satellites broadcasting TDMA signals not originally intended for navigation. The TDMA structure of the carrier phase data introduces carrier phase ambiguities, which can be partially removed via preprocessing based on rough orbit estimates. It is often impossible to remove a residual integer-valued Doppler shift measured in cycles per data burst. The orbit determination square-root information Kalman filter (SRIF) is a mixed real/integer extended Kalman filter which deals explicitly with the integer Doppler shift ambiguities of its partially corrected carrier phase measurements. It isolates the integer unknowns from the real-valued unknowns, and solves for its integer-valued estimates using an integer linear least-squares solver. The carrier phase and pseudorange measurements are available at known measurement times at ground-based receivers, but they provide information about the satellite's orbital state at an unknown signal transmission time. An additional feature of the Kalman filter is its explicit treatment of the uncertain range delay. This is accomplished by applying a new technique that includes Chebyshev polynomial models of random process noise, dense output state interpolation that is consistent with numerical integration of the state differential equation, iterative solution for signal transmission times, and consistent propagation of all of these effects through the SRIF. The effectiveness of this new Kalman filter has been evaluated using truth-model data. The truth model simulation tests evaluate the filter results against known "truth" states and known "truth" Doppler shift ambiguities. The simulation results predict that the Kalman filter will achieve RMS position errors of about XX meters magnitude using only five ground stations located in the contiguous United States.
\end{abstract}

\section{Introduction}

AL manner of orbit determination problems are solved everyday, including post-processing, near real-time Aorbit determination, and orbit predictions. A variety of algorithms are used to produce these estimates of satellite position and velocity. Batch filtering and Kalman filtering are popular methods. Whenever radio navigation data are used, the problem of dealing with implicit measurement times arises. This is not a new problem, however the methods for dealing with the implicit measurement times are not always mathematically rigorous. Recent work, however, has shown how to deal with implicit measurement times within a Kalman filter. ${ }^{1}$

The signals used for orbit determination in this work are L-band TDMA down-link signals, never intended for use in orbit determination. When using TDMA signals, special considerations arise because the signal is not broadcast continuously. Between data bursts, some unknown integer number of carrier cycles elapses, and it is impossible to know exactly how many, because unmodeled accelerations and noise create Doppler shift uncertainty. Through clever preprocessing of the data based on rough orbit estimates, much of the residual ambiguity can be removed, but there often remains some discrete set of possible residual Doppler shift errors. This Doppler shift error can be characterized by an integer number of carrier cycles per data

\footnotetext{
* Graduate Student, Sibley School of Mechanical and Aerospace Engineering, AIAA Student Member

${ }^{\dagger}$ Professor, Sibley School of Mechanical and Aerospace Engineering, AIAA Associate Fellow
}

Copyright (C)2011 by Ryan C. Dougherty and Mark L. Psiaki. All rights reserved.
Preprint for the 2011 AIAA Guidance, Navigation, \& Control Conference 
burst. Figure 1 illustrates how the number of carrier cycles that elapse between data bursts can be uncertain. Note the two example possible carriers in the center of the figure that both connect the broadcast carriers at either end of the figure. They have different numbers of elapsed cycles. The preprocessing mentioned earlier cannot fully remove this uncertainty; instead it guarantees a certain correlation between the integer uncertainties of neighboring interburst periods.

This paper makes two main contributions: one is to adapt the implicit measurement time Kalman filtering techniques of Ref. 1 to this orbit determination problem, the other is to apply the techniques of Ref. 2 in order to directly estimate the TDMA Doppler shift ambiguities as integers. The algorithm of Ref. 1 is applied in a straightforward manner. It involves a generalization of the zero-order-hold process noise assumption to polynomial process noise and the solution of constraints that define the measurement times. This is the first application of these techniques to orbit determination.

Estimation of the TDMA Doppler shift ambiguities gives rise to a mixed real and integer estimation problem.

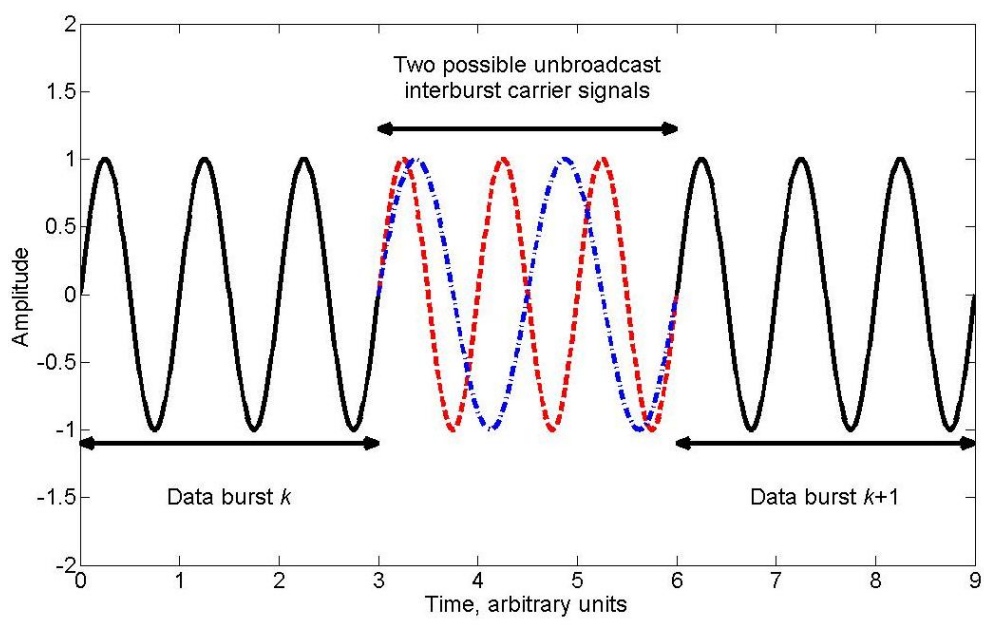

Figure 1. Two successive data bursts (solid lines) and alternative possible interburst carrier waves (dashed and dash-dotted lines). This non-linear estimation problem is linearized using EKF-type methods, and the techniques of Ref. 2 are used to solve the resulting mixed real and integer linear estimation problem.

Another contribution of this paper is its synthesis of the methods in Refs. 1 and 2 into a single algorithm that can handle both implicit measurement times and Doppler shift integer ambiguities.

The remainder of this paper is organized into sections describing the various elements of the overall orbit determination algorithm. Section II defines the state vector and dynamics model used throughout. Section III deals with the measurements and measurement model. It includes discussions of the measurement model components, the measurement constraint equation that implicitly determines the measurement effectiveness time, and the linearization of the measurement equations. The filter is described in Section IV. It is a sampled-data SRIF that is specially tailored to deal with implicit measurement times and mixed real and integer estimation. Section V describes a truth model which was used to evaluate the efficacy of the filter. The paper ends with a short section that summarizes the results of truth model simulations and draws some conclusions.

\section{Dynamics Modeling and Linearization}

\section{A. State Vector Definition}

The state vector used in the present work is broken into four subsets of elements, $\boldsymbol{x}, \boldsymbol{b}_{P}, \boldsymbol{b}_{\phi}$, and $\boldsymbol{n}$. The first subset represents the satellite state and parameters associated with the satellite. The second and third store the real-valued carrier phase and pseudorange biases associated with each pass over each ground station. The fourth stores the integer-valued Doppler shift ambiguity associated with each pass over each ground station.

The first subset, $\boldsymbol{x}$, is composed of thirteen elements, as shown in Eq. (1).

$$
\boldsymbol{x}=\left[\begin{array}{lllllll}
\boldsymbol{r}^{\mathrm{T}} & \boldsymbol{v}^{\mathrm{T}} & \boldsymbol{a}^{\mathrm{T}} & \beta_{d} & \beta_{s} & \delta t & \delta f
\end{array}\right]^{\mathrm{T}}
$$

The first six elements of $\boldsymbol{x}$ are the position vector, $\boldsymbol{r}$, and velocity vector, $\boldsymbol{v}$, of the spacecraft in Earthcentered inertially-fixed (ECIF) coordinates. The seventh through ninth elements, $\boldsymbol{a}$, are the acceleration disturbances in local level coordinates, each of which is modeled by a Markov process. The tenth and eleventh elements are the atmospheric drag inverse ballistic coefficient, $\beta_{d}$, and the inverse solar radiation pressure 
coefficient, $\beta_{s}$; these are also modeled as Markov processes. The last two elements are spacecraft clock error, $\delta t$, and frequency error, $\delta f$, as in Ref. 3 .

The second subset of state elements, $\boldsymbol{b}_{P}$, is a vector of real-valued pseudorange biases. The third subset, $\boldsymbol{b}_{\phi}$, is a vector of real-valued carrier phase biases. Each time a ground station begins tracking a signal from a spacecraft, there is an associated unknown initial spacecraft clock time - the pseudorange bias - and an associated constant, unknown, real-valued initial bias in the measured beat carrier phase. These biases are nuisance parameters and must be estimated along with the rest of the state.

The final subset of the state vector, $\boldsymbol{n}$, is a vector of integer ambiguities, each associated with a particular ground station and satellite pass. These constant integers count the number of carrier cycles that are overor under-counted during the off-time of each TDMA signal per signal burst. They can be thought of as unknown integer Doppler shift biases.

\section{B. Satellite State Dynamics}

The time evolution of the $\boldsymbol{x}$ components of the state vector is modeled by a system of first-order, nonlinear, ordinary differential equations. The spacecraft trajectory is modeled by the summation of various components of a force model. All other spacecraft state elements are modeled as general Markov processes.

The differential equations that describe the satellite trajectory include the effects on the trajectory of higher-order Earth gravity terms, sun and moon gravity gradient, atmospheric drag, solar radiation pressure, and process noise. The Earth gravity model uses EGM96 coefficients in the non-singular recursion II of Table 2 in Ref. 4. This model requires a coordinate transformation from the ECIF frame of $\boldsymbol{r}$ to the EarthCentered Earth-fixed (ECEF) frame of the EGM96 model. This calculation is carried out using International Earth Rotation and Reference System Service Bulletin B information. Sun and moon gravity gradients are calculated using fully non-linear inverse square law gravity gradients and sun and moon locations calculated using the methods of Ref. 5, pp 70-73. Atmospheric drag is calculated using a cannon ball spacecraft model and the Harris-Priester atmospheric density model. ${ }^{5}$ Solar radiation pressure is calculated using the inverse of the square of the actual distance from the sun to the satellite, which causes slight variations over the course of an orbit about the Earth. The calculation of solar radiation pressure also accounts for full and partial occultation of the sun by the Earth.

The differential equations for this model take the form

$$
\begin{aligned}
\dot{\boldsymbol{r}} & =\boldsymbol{v} \\
\dot{\boldsymbol{v}} & =\boldsymbol{g}_{E S M}(t, \boldsymbol{r})+\boldsymbol{a}_{d}\left(t, \boldsymbol{r}, \boldsymbol{v}, \beta_{d}\right)+\boldsymbol{a}_{s}\left(t, \boldsymbol{r}, \beta_{s}\right)+A_{E C I F / L L}(\boldsymbol{r}, \boldsymbol{v}) \boldsymbol{a}
\end{aligned}
$$

where $\boldsymbol{g}_{E S M}$ is the gravitational acceleration due to the Earth, the sun, and the moon, $\boldsymbol{a}_{d}$ is the acceleration due to drag, $\boldsymbol{a}_{s}$ is the acceleration due to solar radiation pressure, $A_{E C I F / L L}$ is the transformation from local level coordinates to ECIF coordinates, and $\boldsymbol{a}$ is the acceleration disturbance vector in local level coordinates.

The acceleration disturbances in local level coordinates are modeled as independent first-order Gauss Markov processes. They each have a time constant $\tau_{a}$ and a steady state acceleration standard deviation $\sigma_{a}$. They evolve according to the differential equation

$$
\dot{\boldsymbol{a}}=\frac{-1}{\tau_{a}} \boldsymbol{a}+\sigma_{a} \sqrt{\frac{2}{\tau_{a}}} \boldsymbol{w}_{a}
$$

where $\boldsymbol{w}_{a}$ is white noise with $\mathrm{E}\left[\boldsymbol{w}_{a}(t)\right]=0$ and $\mathrm{E}\left[\boldsymbol{w}_{a}(t) \boldsymbol{w}_{a}^{\mathrm{T}}(\tau)\right]=I \delta(t-\tau)$.

The drag inverse ballistic coefficient can be used to compute the acceleration due to drag by

$$
\boldsymbol{a}_{d}\left(t, \boldsymbol{r}, \boldsymbol{v}, \beta_{d}\right)=-\frac{1}{2} \beta_{d} \rho(\boldsymbol{r})\left\|\boldsymbol{v}-\omega_{E} \times \boldsymbol{r}\right\|\left[\boldsymbol{v}-\omega_{E} \times \boldsymbol{r}\right]
$$

where $\rho(\boldsymbol{r})$ is the atmospheric density at the spacecraft position, $\omega_{E}$ is the rotation rate of the Earth expressed in ECIF coordinates. The parameter $\beta_{d}$ itself is modeled by the following random walk differential equation

$$
\dot{\beta}_{d}=w_{d}
$$

where $w_{d}$ is white noise with $\mathrm{E}\left[w_{d}\right]=0$ and $\mathrm{E}\left[w_{d}(t) w_{d}^{\mathrm{T}}(\tau)\right]=q_{d} \delta(t-\tau)$. If $q_{d}$ is set to zero, then $\beta_{d}$ is modeled as a constant. 
The solar radiation pressure coefficient can be used to model the solar radiation pressure acceleration with

$$
\boldsymbol{a}_{s}(t, \boldsymbol{r})=-P_{s 0}\left(\boldsymbol{r}_{s}, \boldsymbol{r}\right) \beta_{s} \frac{\left\|\boldsymbol{r}_{s}\right\|^{2}}{\left\|\boldsymbol{r}_{s}-\boldsymbol{r}\right\|^{3}}\left[\boldsymbol{r}_{s}-\boldsymbol{r}\right]
$$

where $P_{s 0}$ is the nominal solar radiation pressure at $1 \mathrm{AU}$ with a scaling to account for occultation of the sun by the Earth, and $\boldsymbol{r}_{s}$ is the position of the sun. The parameter $\beta_{s}$ is also modeled by a random walk:

$$
\dot{\beta}_{s}=w_{s}
$$

where $w_{s}$ is white noise with $\mathrm{E}\left[w_{s}\right]=0$ and $\mathrm{E}\left[w_{s}(t) w_{s}^{\mathrm{T}}(\tau)\right]=q_{s} \delta(t-\tau)$. If $q_{s}$ is set to zero, then $\beta_{s}$ is modeled as a constant.

The last elements of the spacecraft state vector are the clock states. The clock is modeled with two states - the spacecraft clock error and the oscillator frequency error. The oscillator frequency error is modeled as a random walk and the clock error is the integral of the oscillator frequency error plus another random walk.

$$
\left[\begin{array}{c}
\dot{\delta t} \\
\dot{\delta f}
\end{array}\right]=\left[\begin{array}{ll}
0 & 1 \\
0 & 0
\end{array}\right]\left[\begin{array}{l}
\delta t \\
\delta f
\end{array}\right]+\boldsymbol{w}_{c}
$$

As described in Ref. 3, the clock model process noise, $\boldsymbol{w}_{c}$, has statistics

$$
\begin{gathered}
\mathrm{E}\left[\boldsymbol{w}_{c}(t)\right]=0 \\
\mathrm{E}\left[\boldsymbol{w}_{c}(t) \boldsymbol{w}_{c}^{\mathrm{T}}(\tau)\right]=\delta(t-\tau)\left[\begin{array}{cc}
S_{f} & 0 \\
0 & S_{g}
\end{array}\right]
\end{gathered}
$$

where $S_{f}$ and $S_{g}$ are related to the Allan variance parameters by

$$
\begin{gathered}
S_{f} \sim h_{0} / 2 \\
S_{g} \sim 2 \pi^{2} h_{-2}
\end{gathered}
$$

In summary, the satellite state vector model takes the form

$$
\dot{\boldsymbol{x}}(t)=\boldsymbol{f}(t, \boldsymbol{x}(t), \boldsymbol{w}(t))
$$

where the 13 elements of the vector function $\boldsymbol{f}$ have been defined by Eqs. (2)-(4), Eq. (6), Eq. (8), and Eq. (9). The noise vector $\boldsymbol{w}(t)=\left[\boldsymbol{w}_{a}^{\mathrm{T}}(t), w_{d}(t), w_{s}(t), \boldsymbol{w}_{c}^{\mathrm{T}}\right]^{\mathrm{T}}$ has seven elements described in the text following Eq. (4), Eq. (6), Eq. (8), and Eq. (9).

\section{Pseudorange Bias, Carrier Bias, and Doppler Ambiguity Dynamics}

The individual elements of the pseudorange bias vector, $\boldsymbol{b}_{P}$, the carrier phase bias vector, $\boldsymbol{b}_{\phi}$, and the Doppler shift ambiguity vector, $\boldsymbol{n}$, are modeled as constants, but the number of elements of each vector can change over time. In the case of the $\boldsymbol{b}_{P}$ vector, with each new pass over each ground station, one new pseudorange bias is introduced. Thus, one includes the possibility of adding elements during the interval from sample time $t_{k}$ to sample time $t_{k+1}$

$$
\boldsymbol{b}_{P k+1}=\left[\begin{array}{c}
\boldsymbol{b}_{P k} \\
\delta \boldsymbol{b}_{P k+1}
\end{array}\right]
$$

where $\boldsymbol{b}_{P k}$ are the pseudorange biases that were introduced prior to $t_{k}$ and $\delta \boldsymbol{b}_{P k+1}$ are the new pseudorange biases introduced between $t_{k}$ and $t_{k+1}$, and the new pseudorange bias vector, $\boldsymbol{b}_{P k+1}$, at time $t_{k+1}$ is the concatenation of the bias vector at sample time $t_{k}$ and any new biases introduced on the interval between $t_{k}$ and $t_{k+1}$.

Like $\boldsymbol{b}_{P k}, \boldsymbol{b}_{\phi k}$ must be allowed to grow to accommodate new carrier phase biases. Each new pass over each ground station introduces a new bias, so define

$$
\boldsymbol{b}_{\phi k+1}=\left[\begin{array}{c}
\boldsymbol{b}_{\phi k} \\
\delta \boldsymbol{b}_{\phi k+1}
\end{array}\right]
$$


where $\boldsymbol{b}_{\phi k+1}, \boldsymbol{b}_{\phi k}$, and $\delta \boldsymbol{b}_{\phi k+1}$ are defined analogously with the definitions of $\boldsymbol{b}_{P k+1}, \boldsymbol{b}_{P k}$, and $\delta \boldsymbol{b}_{P k+1}$, but they apply to existing and new carrier phase biases.

The Doppler ambiguity vector, $\boldsymbol{n}$, must be able to grow with time for the same reasons. So define

$$
\boldsymbol{n}_{k+1}=\left[\begin{array}{c}
\boldsymbol{n}_{k} \\
\delta \boldsymbol{n}_{k+1}
\end{array}\right]
$$

where $\boldsymbol{n}_{k+1}, \boldsymbol{n}_{k}$, and $\delta \boldsymbol{n}_{k+1}$ are similarly defined, except that they apply to existing and new Doppler shift ambiguities rather than phase or pseudorange biases.

\section{Satellite State Propagation}

By numerically integrating Eq. (10) from time $t_{k}$ to time $t_{k+1}>t_{k}$, given the satellite state at time $t_{k}$ and a continuous description of the process noise over that interval, the satellite state at time $t_{k+1}$ can be found. Considering that the measurement signals are transmitted at some time before they are received, and that the transmission times will have to be solved through an implicit relationship, a continuous description of the satellite state over the interval from time $t_{k}$ to $t_{k+1}$ must be kept. Such a description can take many forms, but the one used herein is an appropriate interpolating polynomial for the Runge-Kutta numerical integration algorithm. These interpolating polynomials are often called dense output Runge-Kutta methods. For more about dense output Runge-Kutta methods, see Refs. 5 and 6.

A continuous description of the process noise is also necessary. See Section II.F for more in-depth coverage of this topic. For now, it suffices to say that the process noise over an integration interval is described continuously by means of a polynomial. In the present work, Chebyshev polynomials describe the process noise over each integration interval. So, each element of the process noise vector over a given interval is represented by the weighted sum of $M+1$ Chebyshev polynomials of order 0 to $M$. The process noise model for integration interval $k$ takes the form,

$$
\boldsymbol{w}_{k}(t)=\sum_{j=0}^{M} \tilde{\boldsymbol{w}}_{k}^{j} C_{j}\left(\eta\left(t, t_{k}\right)\right)
$$

where $C_{j}\left(\eta\left(t, t_{k}\right)\right)$ is the $j^{\text {th }}$ order Chebyshev polynomial of the first kind, $\eta\left(t, t_{k}\right)=\frac{2}{\Delta t}\left(t-t_{k}\right)-1$ is the normalized time argument to the $j^{\text {th }}$ Chebyshev polynomial, $\Delta t$ is a constant defined as $t_{k+1}-t_{k}$ for all $k$, and $\tilde{\boldsymbol{w}}_{k}^{j}$ is the vector of weighting coefficients for the process noise elements of the $j^{\text {th }}$ order polynomial valid over the $k^{\text {th }}$ interval. As in Ref. 1 and described in Section II.F, the coefficients of the process noise polynomials are modeled as a zero-mean white noise sequence such that the process noise is uncorrelated from one interval to all others and optimally approximates the statistics of white noise. Also note that the coefficients $\tilde{\boldsymbol{w}}_{k}^{j}$, though not known a priori, are estimated by the filter as parameters that affect the evolution of the state over the interval.

In summary, the dynamic propagation computes the solution to the differential equation (10) using $\boldsymbol{w}_{k}(t)$ as defined in Eq. (14) and starting at the initial condition $\boldsymbol{x}\left(t_{k}\right)=\boldsymbol{x}_{k}$. Formally, this solution can be written as

$$
\boldsymbol{x}\left(t ; t_{k}, t_{k+1}, \boldsymbol{x}_{k}, \tilde{\boldsymbol{w}}_{k}^{0}, \tilde{\boldsymbol{w}}_{k}^{1}, \ldots, \tilde{\boldsymbol{w}}_{k}^{M}\right)=\boldsymbol{x}\left(t ; \Sigma_{k}\right),
$$

where $\Sigma_{k} \triangleq\left\{t_{k}, t_{k+1}, \boldsymbol{x}_{k}, \tilde{\boldsymbol{w}}_{k}^{0}, \tilde{\boldsymbol{w}}_{k}^{1}, \ldots, \tilde{\boldsymbol{w}}_{k}^{M}\right\}$.

This solution is available for all $t$ in the interval $t_{k}$ to $t_{k+1}$ through use of the Runge-Kutta interpolation. It is needed at measurement times that are to be determined and at the final time $t=t_{k+1}$.

\section{E. Linearized Satellite Dynamics}

The filter described in Section IV is an extended Kalman filter which linearizes the dynamics and measurement model about estimates. The filter requires the partial derivatives of the state with respect to all of the variables needed to propagate the state from time $t_{k}$ to $t_{k+1}$. It also needs partial derivatives at intermediate times. The quantities needed are the state transition matrix,

$$
\Phi_{k}\left(t, t_{k}\right)=\frac{\partial \boldsymbol{x}\left(t ; \Sigma_{k}\right)}{\partial \boldsymbol{x}_{k}},
$$


and the influence matrices of the Chebyshev polynomial process noise coefficients,

$$
\Gamma_{k}^{j}\left(t, t_{k}\right)=\frac{\partial \boldsymbol{x}\left(t ; \Sigma_{k}\right)}{\partial \tilde{\boldsymbol{w}}_{k}^{j}} \text { for } j=0 \text { to } M .
$$

These are determined by Runge-Kutta integration and interpolation of their respective matrix initial value problems. See Ref. 1 for details.

It is convenient to lump the Chebyshev polynomial coefficient vectors into one large discrete-time process noise vector.

$$
\tilde{\boldsymbol{w}}_{k}=\left[\left(\tilde{\boldsymbol{w}}_{k}^{0}\right)^{\mathrm{T}}\left(\tilde{\boldsymbol{w}}_{k}^{1}\right)^{\mathrm{T}} \ldots\left(\tilde{\boldsymbol{w}}_{k}^{M}\right)^{\mathrm{T}}\right]^{\mathrm{T}}
$$

and to define the corresponding influence matrix.

$$
\Gamma_{k}\left(t, t_{k}\right)=\frac{\partial \boldsymbol{x}\left(t ; \Sigma_{k}\right)}{\partial \tilde{\boldsymbol{w}}_{k}}=\left[\Gamma_{k}^{0}\left(t, t_{k}\right) \Gamma_{k}^{1}\left(t, t_{k}\right) \ldots \Gamma_{k}^{M}\left(t, t_{k}\right)\right]
$$

These Jacobian matrices can be used to define linearized dynamics. First, it is helpful to define the point about which linearizations are being made. The process noise linearization point is the a priori process noise estimate, $\tilde{\boldsymbol{w}}_{k}=0$. The model requires a point for state linearization at all possible interpolation times in the interval between sample times $t_{k}$ and $t_{k+1}$. This point is

$$
\boldsymbol{x}\left(t ; \hat{\Sigma}_{k}\right)=\boldsymbol{x}\left(t ; t_{k}, t_{k+1}, \hat{\boldsymbol{x}}_{k}, 0, \ldots, 0\right)
$$

where $\hat{\boldsymbol{x}}_{k}$ is the a posteriori state estimate at sample time $t_{k}$.

The linearized dynamics now take the form

$$
\left[\boldsymbol{x}(t)-\boldsymbol{x}\left(t ; \hat{\Sigma}_{k}\right)\right]=\Phi_{k}\left(t, t_{k}\right)\left[\boldsymbol{x}_{k}-\hat{\boldsymbol{x}}_{k}\right]+\Gamma_{k}\left(t, t_{k}\right) \tilde{\boldsymbol{w}}_{k}
$$

One might be tempted to define $\Delta \boldsymbol{x}_{k}=\boldsymbol{x}-\hat{\boldsymbol{x}}_{k}, \Delta \boldsymbol{x}(t)=\boldsymbol{x}(t)-\overline{\boldsymbol{x}}(t)$, and $\Delta \boldsymbol{x}_{k+1}=\Delta \boldsymbol{x}\left(t_{k+1}\right)$ and use these quantities to simplify the above equation. This is not advisable because the definition of $\Delta \boldsymbol{x}_{k+1}$ would change in going from sample interval $t_{k}$ to $t_{k+1}$ to sample interval $t_{k+1}$ to $t_{k+2}$.

Note the following shorthand notation is used to develop the Kalman filter: $\Phi_{k}=\Phi_{k}\left(t_{k+1}, t_{k}\right), \Gamma_{k}=$ $\Gamma_{k}\left(t_{k+1}, t_{k}\right)$, and $\overline{\boldsymbol{x}}_{k+1}=\boldsymbol{x}\left(t_{k+1} ; \hat{\Sigma}_{k}\right)$. That is, when the time arguments are omitted, the state transition matrix and process noise influence matrix apply to the end of the time interval as does $\overline{\boldsymbol{x}}_{k+1}$. These can be used to write the linearized filter dynamics model for the interval:

$$
\left[\boldsymbol{x}_{k+1}-\overline{\boldsymbol{x}}_{k+1}\right]=\Phi_{k}\left[\boldsymbol{x}_{k}-\hat{\boldsymbol{x}}_{k}\right]+\Gamma_{k} \tilde{\boldsymbol{w}}_{k}
$$

This is just Eq. (21) evaluated at $t=t_{k+1}$.

\section{F. Chebyshev Polynomial Process Noise}

This section briefly reviews the use of Chebyshev polynomials to approximate continuous-time white noise and explains an appropriate statistical model. The material in this section paraphrases Section III.A. of Ref. 1. For additional information on the topics covered in this section, see that paper.

The motivation for using a polynomial description of process noise over an integration interval is a side effect of the numerical integration scheme used. The natural integration interval of the system is too long for zero-order hold process noise to provide a good approximation of white noise over an entire integration interval. It is necessary to use a higher-order approximation of white noise that is consistent with use of implicit measurement times in a filter. Reference 1 shows how this can be done using the Chebyshev expansion of Eq. (14).

In order to do this, it is necessary to define a statistical model for $\tilde{\boldsymbol{w}}_{k}$. The statistical model from Ref. 1 is

$$
\mathrm{E}\left[\tilde{\boldsymbol{w}}_{k}\right]=0 .
$$




$$
\mathrm{E}\left[\tilde{\boldsymbol{w}}_{k}\left(\tilde{\boldsymbol{w}}_{j}\right)^{\mathrm{T}}\right]=\delta_{k j}\left[\begin{array}{cccc}
\alpha_{00} Q & 0 & \ldots & 0 \\
0 & \alpha_{11} Q & \ldots & 0 \\
\vdots & \vdots & \ddots & \vdots \\
0 & 0 & \ldots & \alpha_{M M} Q
\end{array}\right]
$$

where $\alpha_{00}, \alpha_{11}, \ldots, \alpha_{M M}$ are chosen to make the extension of the continuous time process noise model in Eq. (14) to all integration intervals have an autocorrelation that is like the theoretical autocorrelation for true white noise:

$$
\mathrm{E}\left[\boldsymbol{w}(t) \boldsymbol{w}^{\mathrm{T}}(\tau)\right]=Q \delta(t-\tau)
$$

Specific coefficient values $\alpha_{00}, \alpha_{11}, \ldots, \alpha_{M M}$ are determined in Ref. 1 by solving an optimization problem that maximizes the accuracy of this approximation. Several sets of optimal values are listed for several choices of $M$ in Ref. 1 . Note that these coefficients set the covariance matrix of $\tilde{\boldsymbol{w}}$ and thus the a priori information matrix in what follows.

\section{Measurement Modeling and Linearization}

The following sections develop the model that relates the physical measurements used in this work to the state of the system.

\section{A. Measurement Model}

The filter in the present work uses measurements of beat carrier phase and a biased pseudorange measurement. Beat carrier phase is the difference between the phase of the received signal and the phase of the receiver's replica of the nominal signal. Beat carrier phase can be measured very accurately, down to about one fortieth of a cycle. Pseudorange is the range-equivalent time difference between receiver clock time at time of reception and satellite clock time at time of transmission of designated signal features. The arrival time of a signal feature can be measured to within about one microsecond. The satellite clock time at time of transmission of a particular feature is unknown, but the interval between features is regular and known in satellite clock time.

\section{Beat Carrier Phase Measurements}

The following equation explicitly gives the range-equivalent beat carrier phase.

$$
\lambda \phi=\rho+c\left(\tau_{\text {tropo }}-\tau_{\text {iono }}-\left.\delta t\right|_{t_{T}}\right)+b_{\phi j}+\lambda \Delta m n_{i}+v_{\phi}
$$

In this equation, $\lambda$ is the wavelength of the carrier signal, $\phi$ is the beat carrier phase, $c$ is the speed of light in vacuum, $\tau_{\text {tropo }}$ is the propagation delay due to the troposphere, $\tau_{\text {iono }}$ is the phase advance in seconds due to the ionosphere, $\left.\delta t\right|_{t_{T}}$ is the spacecraft clock error at transmission time, $b_{\phi j}$ is the carrier phase bias in meters associated with this measurement, $n_{i}$ is the Doppler shift ambiguity associated with this measurement, $\Delta m$ is the number of data bursts that have elapsed since the initial data burst for this signal was tracked, $v_{\phi}$ is measurement noise, and $\rho$ is the geometrical range between the satellite at time of transmission and the ground station at time of reception, that is,

$$
\rho=\sqrt{\left[\left.\boldsymbol{r}_{R}\right|_{t_{R}}-\left.\boldsymbol{r}\right|_{t_{T}}\right]^{\mathrm{T}}\left[\left.\boldsymbol{r}_{R}\right|_{t_{R}}-\left.\boldsymbol{r}\right|_{t_{T}}\right]}
$$

where $\left.\boldsymbol{r}_{R}\right|_{t_{R}}$ is the location of the receiver at the time of reception and $\left.\boldsymbol{r}\right|_{t_{T}}$ is the location of the satellite at the time of transmission.

The propagation delay due to refractivity of the neutral atmosphere, $\tau_{\text {tropo }}$, is calculated using the Saastamoinen \& Ifadis delay models. ${ }^{7}$ The ionosphere carrier phase advance $\tau_{\text {iono }}=\frac{40.3 \cdot \mathrm{TEC}}{f^{2}}$ is calculated based on TEC from an ionospheric model. In this paper's calculations, the Klobuchar model is used. ${ }^{8}$

The receiver clocks are assumed to be perfectly synchronized to true time. In the truth model simulation, this assumption is enforced. In actual fact, the receiver clock is not perfect, but it is disciplined to GPS time 
using a coupled GPS receiver, and the errors in the receiver clock are much smaller than the errors in the satellite clock.

A new carrier phase bias is added to the $\boldsymbol{b}_{\phi k}$ vector every time a ground station begins tracking a signal from the satellite - once per pass overhead. In Eq. (26), $b_{\phi j}$ is the element of $\boldsymbol{b}_{\phi k+1}$ that applies to the carrier phase bias associated with the current pass of the reference station located at $\boldsymbol{r}_{R}$. The element $b_{\phi j}$ must be appended to the bias vector as part of $\delta \boldsymbol{b}_{\phi k}$ in Eq. (12) for the time interval $t_{k}$ to $t_{k+1}$ if and only if the first measurement sample of the given pass occurs in that time interval. The need for observability of the $\boldsymbol{b}_{\phi}$ vector precludes appending this bias element earlier.

The Doppler shift ambiguity is measured in cycles per burst. This is the number of carrier cycles that are over- or under-counted in the dead time between signal bursts. In a way similar to the carrier phase biases, a new Doppler shift ambiguity is added to the $\boldsymbol{n}_{k}$ vector every time a ground station begins tracking the satellite signal. In Eq. (26), $n_{i}$ is the element of $\boldsymbol{n}_{k+1}$ that applies to the current pass over the reference station located at $\boldsymbol{r}_{R}$. The integer $\Delta m$ starts at 0 for the first measurement of the given pass and increments by one for each subsequent measurement. Thus, $n_{i}$ only affects the second and later measurements of the given pass, that is, when $\Delta m \geq 1$. Therefore, the element $n_{i}$ must be part of the appended set $\delta \boldsymbol{n}_{k}$ from Eq. (13) if and only if the second measurement of the given pass occurs during the time interval $t_{k}$ to $t_{k+1}$. Inclusion at an earlier sample would result in an unobservable element of the ambiguity vector. Figure 2 illustrates the effect of five different estimates of $n_{i}$ on the corresponding beat carrier phase correction as

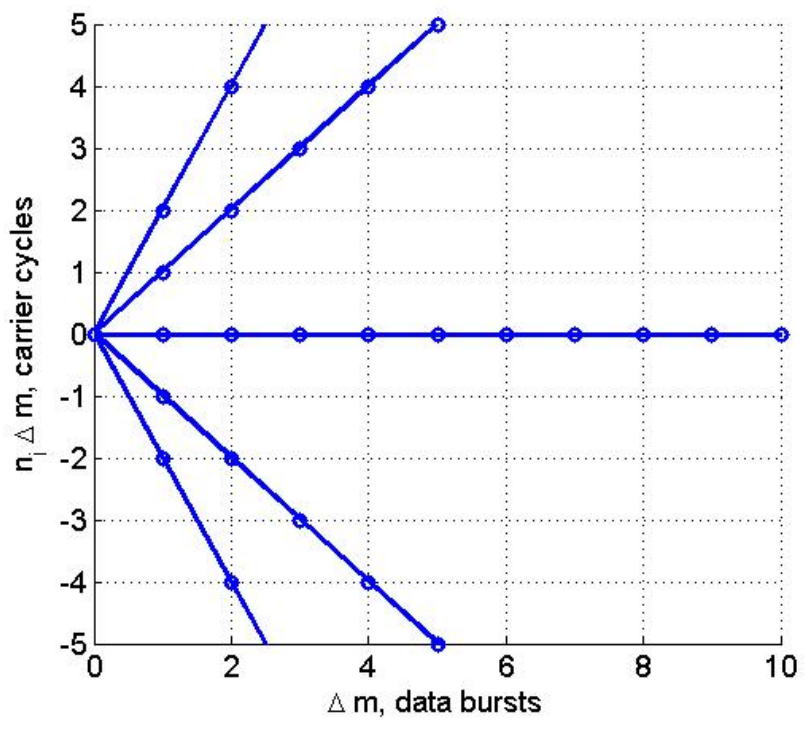

Figure 2. The effects of five possible Doppler shift ambiguities on the beat carrier phase. measured in samples since the beginning of a pass.

Suppose there are $N_{m k}$ beat carrier phase measurements during the time interval from $t_{k}$ to $t_{k+1}$. Each individual measurement's Eq.-(26) model will take the general form

$$
y_{\phi k q}=h_{\phi k q}\left(t_{R k q}, t_{T k q}, \boldsymbol{b}_{\phi k+1}, \boldsymbol{x}\left(t_{T k q}, \Sigma_{k}\right), \boldsymbol{n}_{k+1}\right)+v_{\phi k q}
$$

where the subscript $q$ indicates the measurement within the sample interval, so that $q$ can range from 1 to $N_{m k}$, the subscript $k$ specifies the sample interval, $t_{R k q}$ is the known reception time, $t_{T k q}$ is the unknown transmission time, and $v_{\phi k q}$ is measurement noise. The subscripts $k q$ on the measurement function are used to determine which reference station appears as $r_{R}$ in Eq. (27) along with the correct indices $j$ and $i$ into $\boldsymbol{b}_{\phi k+1}$ and $\boldsymbol{n}_{k+1}$ to find $b_{\phi j}$ and $n_{i}$.

\section{Biased Pseudorange Measurements}

Pseudorange, $P$, is the apparent range between the receiver at time of reception according to the receiver clock and the satellite at time of transmission according to the satellite clock. In the system under consideration, receiver clock time is available, but satellite clock time is unknown. However, the spacecraft sends signals at exactly regular intervals according to its own imperfect clock. So, if one denotes satellite clock time by $T$, one can write

$$
T+v_{P}=T_{0}+v_{P 0}+\Delta m \Delta T
$$

where $T_{0}$ is some unknown initial spacecraft clock time, $\Delta T$ is the known data burst period which is constant in spacecraft clock time, and $v_{P}$ and $v_{P 0}$ are the noise associated with identifying the received signal feature at the current epoch and at the zeroth epoch. The pseudorange, $P$, is then

$$
P=c\left(t_{R}-\left(T_{0}+v_{P 0}+\Delta m \Delta T-v_{P}\right)\right)
$$


The apparent transit time can be broken into parts due to actual transit time and clock errors. The geometrical range, $\rho$, will contribute to the actual transit time. The signal will experience delays due to passing through the troposphere and ionosphere. These delays are the same as in Section III.A.1, except that one uses $\tau_{\text {iono }}$ with the opposite sign, because the ionospheric phase advance is of equal magnitude and opposite sign as the ionospheric group delay. The apparent transit time is also affected by the receiver clock error at time of reception and the satellite clock error at time of transmission. As stated earlier, the receiver clock error is assumed to be zero at all times, but the satellite clock will have some error, $\left.\delta\right|_{t_{T}}$, at the time of signal transmission. The pseudorange can be written in terms of these apparent transit time components as

$$
P=\rho+c\left(\tau_{\text {tropo }}+\tau_{\text {iono }}-\left.\delta\right|_{t_{T}}\right)
$$

Equating Eq. 30 with Eq. 31 and rearranging, we have

$$
c\left(t_{R}-\Delta m \Delta T\right)=\rho+c\left(\tau_{\text {tropo }}+\tau_{\text {iono }}+T_{0}+v_{P 0}-\left.\delta t\right|_{t_{T}}-v_{P}\right)
$$

The left-hand side of Eq. 32 contains only measured or known quantities. The right-hand side of Eq. 32 contains quantities that depend on the filter states and quantities that can be calculated a priori, with the exception of the constant-over-one-pass quantity $T_{0}+v_{0}$. This quantity is the initial spacecraft clock time with associated measurement error, and it acts as a bias in the pseudorange measurements. Define

$$
b_{P}=T_{0}+v_{0}
$$

and the biased pseudorange measurement can be written explicitly as

$$
c\left(t_{R}-\Delta m \Delta T\right)=\rho+c\left(\tau_{\text {tropo }}+\tau_{\text {iono }}+b_{P l}-\left.\delta\right|_{t_{T}}-v_{P}\right)
$$

As with carrier phase biases, a new pseudorange bias is added to the bias vector, $\boldsymbol{b}_{P k}$, at the beginning of each pass of the satellite over each ground station. In Eq. (34), $b_{P l}$ is the particular element of $\boldsymbol{b}_{P k}$ associated with the current pass over the ground station at $\boldsymbol{r}_{R}$.

Also like the phase biases, the element $b_{P l}$ must be appended to the pseudorange bias vector as an element of $\delta \boldsymbol{b}_{P k}$ in Eq. (11) if and only if the first measurement sample of the current pass occurs in the interval between $t_{k}$ and $t_{k+1}$.

The general form of the biased pseudorange measurement equation will take a form analogous to Eq. (28). Each biased pseudorange measurement on the interval from $t_{k}$ to $t_{k+1}$ will have an Eq.-(34) model of the form

$$
y_{P k q}=h_{P k q}\left(t_{R k q}, t_{T k q}, \boldsymbol{b}_{P k+1}, \boldsymbol{x}\left(t_{T k q}, \Sigma_{k}\right)\right)+v_{P k q}
$$

where all symbols are defined as before, and the subscripts $k q$ perform a similar task to the same subscripts in Eq. (28), they choose which reference station appears as $\boldsymbol{r}_{R}$ in Eq. (27) and the correct index $l$ into $\boldsymbol{b}_{P k+1}$ to find $b_{P l}$.

The physical measurements of the system are related to the satellite state at the time of signal transmission through Eq. (28) and Eq. (35), but the time of signal transmission is still unknown.

\section{B. Measurement Constraint}

The unknown transmission time in the measurement model of Eq. (28) and Eq. (35) is determined from the known reception time $t_{R}$ and the transmission delay. The transmission delay relationship can be written in the form of the following constraint.

$$
0=c\left[t_{R}-t_{T}\right]-\sqrt{\left[\left.\boldsymbol{r}_{R}\right|_{t_{R}}-\left.\boldsymbol{r}\right|_{t_{T}}\right]^{\mathrm{T}}\left[\left.\boldsymbol{r}_{R}\right|_{t_{R}}-\left.\boldsymbol{r}\right|_{t_{T}}\right]}-c\left(\tau_{\text {iono }}+\tau_{\text {tropo }}\right)
$$

where $\tau_{\text {iono }}$, again represents the ionospheric group delay. The time of transmission, $t_{T}$, is computed via a numerical solution of this equation. This numerical solution relies on knowing the function $\boldsymbol{r}(t)$ at various times. This knowledge is provided by the Runge-Kutta state interpolation $\boldsymbol{x}\left(t ; \Sigma_{k}\right)$.

For each of the $N_{m k}$ beat carrier phase measurements and $N_{m k}$ biased pseudorange measurements, there will be a corresponding constraint equation that will take the general form

$$
0=g_{k q}\left(t_{R k q}, t_{T k q}, \boldsymbol{x}\left(t_{T k q}, \Sigma_{k}\right)\right)
$$

where the subscripts $k q$ on $g$ perform analogous tasks as before; that is, they choose the correct ground station to appear as $\boldsymbol{r}_{R}$ in Eq. (36). Note the solution of this constraint for $t_{T k q}$ makes $t_{T k q}$ an implicit function of the elements of $\Sigma_{k}$, e.g., $\boldsymbol{x}_{k}$ and $\tilde{\boldsymbol{w}}_{k}$. 


\section{Linearized Measurement Equations}

The filter developed in Section IV requires a linearized measurement model. In order to develop the linearized measurement model, derivatives of $h_{\phi k q}\left(t_{R k q}, t_{T k q}, \boldsymbol{b}_{\phi k+1}, \boldsymbol{x}\left(t_{T k q}, \Sigma_{k}\right), \boldsymbol{n}_{k+1}\right)$ and derivatives of $h_{P k q}\left(t_{R k q}, t_{T k q}, \boldsymbol{b}_{P k+1}, \boldsymbol{x}\left(t_{T k q}, \Sigma_{k}\right)\right)$ must be computed. In particular, derivatives with respect to the vectors $\boldsymbol{b}_{\phi k+1}, \boldsymbol{b}_{P k+1}, \boldsymbol{n}_{k+1}, \boldsymbol{x}_{k}$, and $\tilde{\boldsymbol{w}}_{k}$ are necessary.

In naming the partial derivatives in Eq. (38)-Eq. (42), the first subscript indicates which measurement function is being differentiated - $\phi$ for carrier phase and $P$ for pseudorange - the second subscript indicates with respect to which quantity it is being differentiated, and the third and fourth subscripts indicate the time step and sample number. When differentiating with respect to biases, the bias-type subscript has been omitted; it is to be understood that differentiation is carried out with respect to the corresponding type of bias. The partial derivatives of the carrier phase measurement function with respect to $\boldsymbol{b}_{\phi k+1}$ and $\boldsymbol{n}_{k+1}$ are straightforward to write for the $q^{\text {th }}$ measurement on sample interval $t_{k}$ to $t_{k+1}$.

$$
\begin{aligned}
& H_{\phi b k q}=\frac{\partial h_{\phi k q}\left(t_{R k q}, t_{T k q}, \boldsymbol{b}_{\phi k+1}, \boldsymbol{x}\left(t_{T k q}, \Sigma_{k}\right), \boldsymbol{n}_{k+1}\right)}{\partial \boldsymbol{b}_{\phi k+1}} \\
& H_{\phi n k q}=\frac{\partial h_{\phi k q}\left(t_{R k q}, t_{T k q}, \boldsymbol{b}_{\phi k+1}, \boldsymbol{x}\left(t_{T k q}, \Sigma_{k}\right), \boldsymbol{n}_{k+1}\right)}{\partial \boldsymbol{n}_{k+1}}
\end{aligned}
$$

Likewise, the partial derivative of the pseudorange measurement function with respect to $\boldsymbol{b}_{P k+1}$ for the $q^{\text {th }}$ pseudorange measurement on the same interval is also easy to find.

$$
H_{P b k q}=\frac{\partial h_{P k q}\left(t_{R k q}, t_{T k q}, \boldsymbol{b}_{P k+1}, \boldsymbol{x}\left(t_{T k q}, \Sigma_{k}\right)\right)}{\partial \boldsymbol{b}_{P k+1}}
$$

These three derivatives can be found by analytically differentiating the respective measurement functions.

The derivatives of the measurement function with respect to $\boldsymbol{x}_{k}$ and $\tilde{\boldsymbol{w}}_{k}$ must be computed using the chain rule of differentiation, because the functions

$$
h_{\phi k q}\left(t_{R k q}, t_{T k q}, \boldsymbol{b}_{\phi k+1}, \boldsymbol{x}\left(t_{T k q}, \Sigma_{k}\right), \boldsymbol{n}_{k+1}\right)
$$

and

$$
h_{P k q}\left(t_{R k q}, t_{T k q}, \boldsymbol{b}_{P k+1}, \boldsymbol{x}\left(t_{T k q}, \Sigma_{k}\right)\right)
$$

depend only indirectly upon $\boldsymbol{x}_{k}$ and $\tilde{\boldsymbol{w}}_{k}$ through $\boldsymbol{x}\left(t_{T k q}, \Sigma_{k}\right)$ and through $t_{T k q}$. The derivatives of the carrier phase measurement function with respect to $\boldsymbol{x}_{k}$ and $\tilde{\boldsymbol{w}}_{k}$ for the $q^{\text {th }}$ measurement on sample interval $t_{k}$ to $t_{k+1}$ are written ${ }^{1}$

$$
\begin{array}{r}
H_{\phi x k q}=\frac{\partial h_{\phi k q}\left(t_{R k q}, t_{T k q}, \boldsymbol{b}_{\phi k+1}, \boldsymbol{x}\left(t_{T k q} ; \Sigma_{k}\right), \boldsymbol{n}_{k+1}\right)}{\partial \boldsymbol{x}\left(t_{T k q} ; \Sigma_{k}\right)}\left[\frac{\partial \boldsymbol{x}\left(t_{T k q} ; \Sigma_{k}\right)}{\partial \boldsymbol{x}_{k}}+\frac{\partial \boldsymbol{x}\left(t_{T k q} ; \Sigma_{k}\right)}{\partial t_{T k q}} \frac{\partial t_{T k q}}{\partial \boldsymbol{x}_{k}}\right] \\
+\frac{\partial h_{\phi k q}\left(t_{R k q}, t_{T k q}, \boldsymbol{b}_{\phi k+1}, \boldsymbol{x}\left(t_{T k q} ; \Sigma_{k}\right), \boldsymbol{n}_{k+1}\right)}{\partial t_{T k q}} \frac{\partial t_{T k q}}{\partial \boldsymbol{x}_{k}} \\
H_{\phi \tilde{w} k q}=\frac{\partial h_{\phi k q}\left(t_{R k q}, t_{T k q}, \boldsymbol{b}_{\phi k+1}, \boldsymbol{x}\left(t_{T k q} ; \Sigma_{k}\right), \boldsymbol{n}_{k+1}\right)}{\partial \boldsymbol{x}\left(t_{T k q} ; \Sigma_{k}\right)}\left[\frac{\partial \boldsymbol{x}\left(t_{T k q} ; \Sigma_{k}\right)}{\partial \tilde{\boldsymbol{w}}_{k}}+\frac{\partial \boldsymbol{x}\left(t_{T k q} ; \Sigma_{k}\right)}{\partial t_{T k q}} \frac{\partial t_{T k q}}{\partial \tilde{\boldsymbol{w}}_{k}}\right] \\
+\frac{\partial h_{\phi k q}\left(t_{R k q}, t_{T k q}, \boldsymbol{b}_{\phi k+1}, \boldsymbol{x}\left(t_{T k q} ; \Sigma_{k}\right), \boldsymbol{n}_{k+1}\right)}{\partial t_{T k q}} \frac{\partial t_{T k q}}{\partial \tilde{\boldsymbol{w}}_{k}}
\end{array}
$$

In both Eq. (41) and Eq. (42) above, the $h_{\phi k q}$ partial derivative in each of the two main terms on the right-hand side can be found by taking the analytical derivative of the measurement function. The leftmost partial derivatives inside the square braces in both equations are derivatives already defined in Eq. (16) and Eq. (19). The first partial derivative in the second term of the expression in square braces in each equation is the time derivative of the state. This quantity can, in general, be obtained in two ways: either by evaluating Eq. (10) with the proper arguments, or by differentiating the interpolating polynomial that is the output of the dense-output Runge-Kutta numerical integration. The former method is used in the present work. 
The partial derivatives of $t_{T k q}$ with respect to $\boldsymbol{x}_{k}$ and $\tilde{\boldsymbol{w}}_{k}$ remain to be determined. The former appears in two places in Eq. (41), the latter in two places in Eq. (42). These are determined via partial differentiation of the constraint that implicitly defines $t_{T k q}$, the constraint in Eq. (37). The derivative with respect to $\boldsymbol{x}_{k}$ is determined by solving the following differentiated form of that constraint.

$$
\begin{aligned}
0=\frac{\partial g_{k q}\left(t_{R k q}, t_{T k q}, \boldsymbol{x}\left(t_{T k q}, \Sigma_{k}\right)\right)}{\partial \boldsymbol{x}\left(t_{T k q} ; \Sigma_{k}\right)}\left[\frac{\partial \boldsymbol{x}\left(t_{T k q} ; \Sigma_{k}\right)}{\partial \boldsymbol{x}_{k}}+\frac{\partial \boldsymbol{x}\left(t_{T k q} ; \Sigma_{k}\right)}{\partial t_{T k q}} \frac{\partial t_{T k q}}{\partial \boldsymbol{x}_{k}}\right] \\
+\frac{\partial g_{k q}\left(t_{R k q}, t_{T k q}, \boldsymbol{x}\left(t_{T k q}, \Sigma_{k}\right)\right)}{\partial t_{T k q}} \frac{\partial t_{T k q}}{\partial \boldsymbol{x}_{k}}
\end{aligned}
$$

Notice that the unknown term $\partial t_{T k q} / \partial \boldsymbol{x}_{k}$ enters linearly and that all other terms are either known already or can be found by taking analytical derivatives of the constraint equation. The solution of Eq. (43) can be substituted into Eq. (41) to complete the definition of $H_{\phi x k q}$. A similar procedure is used to compute $\partial t_{T k q} / \partial \tilde{\boldsymbol{w}}_{k}$ for substitution into Eq. (42). The resulting differentiated constraint varies from Eq. (43) in that all partial derivatives with respect to $\boldsymbol{x}_{k}$ change to partials with respect to $\tilde{\boldsymbol{w}}_{k}$. See Ref. 1 for the original derivation of this method and for more details.

The derivatives $H_{P x k q}$ and $H_{P \tilde{w} k q}$ are found using the same procedure on $h_{P k q}(\ldots)$ and the same differentiated constraint equation.

With the above derivatives, one can write the linearized measurement equation for the $q^{\text {th }}$ measurement on the time interval $t_{k}$ to $t_{k+1}$. Before proceeding, it is useful to define the points about which the measurement equation is linearized. The measurement equation is linearized about the a priori process noise estimate $\overline{\tilde{w}}_{k}=0$, about the a priori pseudorange bias estimate

$$
\overline{\boldsymbol{b}}_{P k+1}=\left[\begin{array}{c}
\hat{\boldsymbol{b}}_{P k} \\
0
\end{array}\right]
$$

about the a priori beat carrier phase bias estimate

$$
\overline{\boldsymbol{b}}_{\phi k+1}=\left[\begin{array}{c}
\hat{\boldsymbol{b}}_{\phi k} \\
0
\end{array}\right]
$$

about the a priori Doppler shift ambiguity estimate

$$
\overline{\boldsymbol{n}}_{k+1}=\left[\begin{array}{c}
\hat{\boldsymbol{n}}_{k} \\
0
\end{array}\right]
$$

and about the same satellite state point about which the dynamics are linearized, $\hat{\boldsymbol{x}}_{k}$. The circumflexes $\left({ }^{\wedge}\right)$ indicate the a posteriori estimates at sample $k$, and the macrons $\left({ }^{-}\right)$indicate the a priori estimates at sample $k+1$. Now, the linearized carrier phase measurement equation for the $q^{\text {th }}$ measurement on the time interval $t_{k}$ to $t_{k+1}$ is

$$
\begin{aligned}
{\left[y_{\phi k q}-h_{\phi k q}\left(t_{R k q}, t_{T k q}, \boldsymbol{b}_{\phi k+1}, \overline{\boldsymbol{x}}\left(t_{T k q} ; \hat{\Sigma}_{k}\right), \boldsymbol{n}_{k+1}\right)\right]=} & H_{\phi \tilde{w} k q} \tilde{\boldsymbol{w}}_{k}+H_{\phi b_{\phi} k q}\left[\boldsymbol{b}_{\phi k+1}-\overline{\boldsymbol{b}}_{\phi k+1}\right] \\
& +H_{\phi x k q}\left[\boldsymbol{x}_{k}-\hat{\boldsymbol{x}}_{k}\right]+H_{\phi n k q}\left[\boldsymbol{n}_{k+1}-\overline{\boldsymbol{n}}_{k+1}\right]+v_{\phi k q}
\end{aligned}
$$

and the linearized pseudorange measurement equation for the $q^{\text {th }}$ pseudorange measurement on the same interval is

$$
\begin{array}{r}
{\left[y_{P k q}-h_{P k q}\left(t_{R k q}, t_{T k q}, \boldsymbol{b}_{P k+1}, \overline{\boldsymbol{x}}\left(t_{T k q} ; \hat{\Sigma}_{k}\right)\right)\right]=H_{P \tilde{w} k q} \tilde{\boldsymbol{w}}_{k}+H_{P b_{P} k q}\left[\boldsymbol{b}_{P k+1}-\overline{\boldsymbol{b}}_{P k+1}\right]} \\
+H_{P x k q}\left[\boldsymbol{x}_{k}-\hat{\boldsymbol{x}}_{k}\right]+v_{P k q}
\end{array}
$$

With one additional definition, all $2 N_{m k}$ measurements in sample interval $t_{k}$ to $t_{k+1}$ can be stacked into a single matrix-vector equation. Let $\Delta y_{\square k q} \triangleq y_{\square k q}-h_{\square k q}(\ldots)$, where $\square$ is either $P$ or $\phi$. Now all $2 N_{m k}$ 
equations can be stacked.

$$
\left[\begin{array}{c}
\Delta y_{\phi k 1} \\
\Delta y_{\phi k 2} \\
\vdots \\
\Delta y_{\phi k N_{m k}} \\
\Delta y_{P k 1} \\
\Delta y_{P k 2} \\
\vdots \\
\Delta y_{P k N_{m k}}
\end{array}\right]=\left[\begin{array}{ccccc}
H_{\phi \tilde{w} k 1} & 0 & H_{\phi b k 1} & H_{\phi x k 1} & H_{\phi n k 1} \\
H_{\phi \tilde{w} k 2} & 0 & H_{\phi b k 2} & H_{\phi x k 2} & H_{\phi n k 2} \\
\vdots & \vdots & \vdots & \vdots & \vdots \\
H_{\phi \tilde{w} k N_{m k}} & 0 & H_{\phi b k N_{m k}} & H_{\phi x k N_{m k}} & H_{\phi n k N_{m k}} \\
H_{P \tilde{w} k 1} & H_{P b k 1} & 0 & H_{P x k 1} & 0 \\
H_{P \tilde{w} k 2} & H_{P b k 2} & 0 & H_{P x k 2} & 0 \\
\vdots & \vdots & \vdots & \vdots & \vdots \\
H_{P \tilde{w} k N_{m k}} & H_{P b k N_{m k}} & 0 & H_{P x k N_{m k}} & 0
\end{array}\right]\left[\begin{array}{c}
\tilde{w}_{k} \\
\boldsymbol{b}_{P k+1}-\overline{\boldsymbol{b}}_{P k+1} \\
\boldsymbol{b}_{\phi k+1}-\overline{\boldsymbol{b}}_{\phi k+1} \\
\boldsymbol{x}_{k}-\hat{\boldsymbol{x}}_{k} \\
\boldsymbol{n}_{k+1}-\overline{\boldsymbol{n}}_{k+1}
\end{array}\right]+\left[\begin{array}{c}
v_{\phi k 1} \\
v_{\phi k 2} \\
\vdots \\
v_{\phi k N_{m k}} \\
v_{P k 1} \\
v_{P k 2} \\
\vdots \\
v_{P k N_{m k}}
\end{array}\right]
$$

Finally, by combining each column in the above equation into a single vector or matrix, one arrives at the final form of the linearized measurement vector equation.

$$
\Delta \boldsymbol{y}_{k+1}=\left[\begin{array}{lllll}
H_{\tilde{w} k} & H_{P k+1} & H_{\phi k+1} & H_{x k} & H_{n k+1}
\end{array}\right]\left[\begin{array}{c}
\tilde{w}_{k} \\
\boldsymbol{b}_{P k+1}-\overline{\boldsymbol{b}}_{P k+1} \\
\boldsymbol{b}_{\phi k+1}-\overline{\boldsymbol{b}}_{\phi k+1} \\
\boldsymbol{x}_{k}-\hat{\boldsymbol{x}}_{k} \\
\boldsymbol{n}_{k+1}-\overline{\boldsymbol{n}}_{k+1}
\end{array}\right]+\boldsymbol{v}_{k}
$$

The row vectors that comprise the large block matrix in Eq. (49) are distinguished notationally from the matrices $H_{\tilde{w} k}, H_{P k+1}, H_{\phi k+1}, H_{x k}$, and $H_{n k+1}$ in Eq. (50) only by two notational details. First, the former retain the sample number subscript, while the latter do not. And, second, the former carry a leading $\phi$ or $P$ subscript and, if applicable, a $b$ subscript, while the latter have dropped their leading $\phi$ or $P$ subscript, except in the second and third block columns of Eq. (50), in which the $\phi$ and $P$ subscripts were kept in favor of the $b$ subscript. Of course, the former are the rows of their respective counterparts in the latter. The sample indices have been incremented by one when stacking the row vectors $H_{P b k q}, H_{\phi b k q}$, and $H_{\phi n k q}$ into the matrices $H_{P k+1}, H_{\phi k+1}$, and $H_{n k+1}$. This was done to improve consistency in Eq. (50) and in what follows. Finally, with the linearized dynamics equation, Eq. (22), and the linearized measurement equation, Eq. (50), one can derive the square-root information extended Kalman filter.

\section{Square-Root Information Extended Kalman Filter}

\section{A. Square-Root Information Formulation}

A square-root information equation stores estimates of the real- and integer-valued states, along with their associated uncertainties. The a posteriori square-root information equations used by the present filter are

$$
\left[\begin{array}{cccc}
\hat{R}_{P P k} & \hat{R}_{P \phi k} & \hat{R}_{P x k} & \hat{R}_{P n k} \\
0 & \hat{R}_{\phi \phi k} & \hat{R}_{\phi x k} & \hat{R}_{\phi n k} \\
0 & 0 & \hat{R}_{x x k} & \hat{R}_{x n k} \\
0 & 0 & 0 & \hat{R}_{n n k}
\end{array}\right]\left[\begin{array}{c}
\boldsymbol{b}_{P k}-\hat{\boldsymbol{b}}_{P k} \\
\boldsymbol{b}_{\phi k}-\hat{\boldsymbol{b}}_{\phi k} \\
\boldsymbol{x}_{k}-\hat{\boldsymbol{x}}_{k} \\
\boldsymbol{n}_{k}-\hat{\boldsymbol{n}}_{k}
\end{array}\right]=\left[\begin{array}{c}
0 \\
0 \\
0 \\
\Delta \hat{\boldsymbol{z}}_{n k}
\end{array}\right]-\left[\begin{array}{c}
\boldsymbol{v}_{P k} \\
\boldsymbol{v}_{\phi k} \\
\boldsymbol{v}_{x k} \\
\boldsymbol{v}_{n k}
\end{array}\right]
$$

The a posteriori information is stored in the square-root information matrices which comprise the large block matrix on the left-hand side of Eq. (51), in the a posteriori estimates, $\hat{\boldsymbol{b}}_{P k}, \hat{\boldsymbol{b}}_{\phi k}, \hat{\boldsymbol{x}}_{k}$, and $\hat{\boldsymbol{n}}_{k}$, and in the information vector $\Delta \hat{\boldsymbol{z}}_{n k}$. The block, upper-triangular, square-root information matrix on the left-hand side is the inverse square-root of the a posteriori covariance matrix of the vector that multiplies it. The white noise vectors, $\boldsymbol{v}_{P k}, \boldsymbol{v}_{\phi k}, \boldsymbol{v}_{x k}$, and $\boldsymbol{v}_{n k}$ are independent, zero-mean, identity-covariance white noise sequences. Notice that only the last of the four equations has a non-homogeneous term, $\Delta \hat{\boldsymbol{z}}_{n k}$, on the right-hand side. This is because the real-valued a posteriori estimates, $\hat{\boldsymbol{b}}_{P k}, \hat{\boldsymbol{b}}_{\phi k}$, and $\hat{\boldsymbol{x}}_{k}$, have been chosen to make their corresponding information equations homogeneous. The optimal estimate $\hat{\boldsymbol{n}}_{k}$ is chosen to minimize $\Delta \hat{\boldsymbol{z}}_{n k}^{\mathrm{T}} \Delta \hat{\boldsymbol{z}}_{n k}$, but it cannot drive this cost metric to zero because it is restricted to take on integer values. Note, a traditional SRIF, as in Ref. 9, does not have the $\hat{\boldsymbol{b}}_{P k}, \hat{\boldsymbol{b}}_{\phi k}, \hat{\boldsymbol{x}}_{k}$, and $\hat{\boldsymbol{n}}_{k}$ terms that appear in 
Eq. (51). Instead, all three equations have non-homogeneous terms. The nonstandard form of Eq. (51) is useful in the context of extended SRIF for nonlinear problems.

The presence of process noise in Eq. (50) makes it more computationally efficient for the SRIF to perform a combined dynamic propagation and measurement update than to perform each separately. The first step forms a set of equations that combines an a priori process noise equation with the a posteriori information equations, Eq. (51), and the measurement equations, Eq. (50), in that order.

$$
\left[\begin{array}{ccccc}
R_{\tilde{w} \tilde{w} k} & {[0,0]} & {[0,0]} & 0 & {[0,0]} \\
0 & {\left[\hat{R}_{P P k}, 0\right]} & {\left[\hat{R}_{P \phi k}, 0\right]} & \hat{R}_{P x k}\left[\hat{R}_{P n k}, 0\right] \\
0 & {[0,0]} & {\left[\hat{R}_{\phi \phi k}, 0\right]} & \hat{R}_{\phi x k} & {\left[\hat{R}_{\phi n k}, 0\right]} \\
0 & {[0,0]} & {[0,0]} & \hat{R}_{x x k} & {\left[\hat{R}_{x n k}, 0\right]} \\
0 & {[0,0]} & {[0,0]} & 0 & {\left[\hat{R}_{n n k}, 0\right]} \\
H_{\tilde{w}_{k}} & H_{P k+1} & H_{\phi k+1} & H_{x k} & H_{n k+1}
\end{array}\right]\left[\begin{array}{c}
\tilde{\boldsymbol{w}}_{k} \\
\boldsymbol{b}_{P k+1}-\hat{\boldsymbol{b}}_{P k+1} \\
\boldsymbol{b}_{\phi k+1}-\hat{\boldsymbol{b}}_{\phi k+1} \\
\boldsymbol{x}_{k}-\hat{\boldsymbol{x}}_{k} \\
\boldsymbol{n}_{k+1}-\overline{\boldsymbol{n}}_{k+1}
\end{array}\right]=\left[\begin{array}{c}
0 \\
0 \\
0 \\
0 \\
\Delta \hat{\boldsymbol{z}}_{n k} \\
\Delta \boldsymbol{y}_{k+1}
\end{array}\right]-\left[\begin{array}{c}
\boldsymbol{v}_{\tilde{w} k} \\
\boldsymbol{v}_{P k} \\
\boldsymbol{v}_{\phi k} \\
\boldsymbol{v}_{x k} \\
\boldsymbol{v}_{n k} \\
\boldsymbol{v}_{k}
\end{array}\right]
$$

where $R_{\tilde{w} \tilde{w} k}$ is the process noise square-root information matrix, which is the inverse square root of the covariance matrix given in Eq. (24). In combining Eq. (50) and Eq. (51), Eq. (51) has been re-written in terms of $\boldsymbol{b}_{P k+1}, \boldsymbol{b}_{\phi k+1}$, and $\boldsymbol{n}_{k+1}$. This was accomplished by noting that $\boldsymbol{b}_{P k+1}, \boldsymbol{b}_{\phi k+1}$, and $\boldsymbol{n}_{k+1}$ equal, respectively, $\boldsymbol{b}_{P k}, \boldsymbol{b}_{\phi k}$, and $\boldsymbol{n}_{k}$, but possibly with additional elements as in Eq. (11), Eq. (12), and Eq. (13). The filter has no a priori information about the added elements, which is accounted for with the additional columns of zeros in the second, third, and fifth block columns of the block matrix on the left-hand side of Eq. (52).

The second step in performing the combined propagation and update is to write the above equation in terms of $\boldsymbol{x}_{k+1}-\overline{\boldsymbol{x}}_{k+1}$. If the linearized dynamics equation, Eq. (22), is solved for $\left[\boldsymbol{x}_{k}-\hat{\boldsymbol{x}}_{k}\right]$ in terms of $\boldsymbol{x}_{k+1}-\overline{\boldsymbol{x}}_{k+1}$ and $\tilde{\boldsymbol{w}}_{k}$, the first and fourth columns of the large block matrix change so that Eq. (52) becomes

$$
\left[\begin{array}{ccccc}
R_{\tilde{w} \tilde{w} k} & {[0,0]} & {[0,0]} & 0 & {[0,0]} \\
-\hat{R}_{P x k} \Phi_{k}^{-1} \Gamma_{k} & {\left[\hat{R}_{P P k}, 0\right]} & {\left[\hat{R}_{P \phi k}, 0\right]} & \hat{R}_{P x k} \Phi_{k}^{-1} & {\left[\hat{R}_{P n k}, 0\right]} \\
-\hat{R}_{\phi x k} \Phi_{k}^{-1} \Gamma_{k} & {[0,0]} & {\left[\hat{R}_{\phi \phi k}, 0\right]} & \hat{R}_{\phi x k} \Phi_{k}^{-1} & {\left[\hat{R}_{\phi n k}, 0\right]} \\
-\hat{R}_{x x k} \Phi_{k}^{-1} \Gamma_{k} & {[0,0]} & {[0,0]} & \hat{R}_{x x k} \Phi_{k}^{-1} & {\left[\hat{R}_{x n k}, 0\right]} \\
0 & {[0,0]} & {[0,0]} & 0 & {\left[\hat{R}_{n n k}, 0\right]} \\
H_{\tilde{w} k}-H_{x k} \Phi_{k}^{-1} \Gamma_{k} & H_{P k+1} & H_{\phi k+1} & H_{x k} \Phi_{k}^{-1} & H_{n k+1}
\end{array}\right]\left[\begin{array}{c}
\tilde{\boldsymbol{w}}_{k} \\
\boldsymbol{b}_{P k+1}-\overline{\boldsymbol{b}}_{P k+1} \\
\boldsymbol{b}_{\phi k+1}-\overline{\boldsymbol{b}}_{\phi k+1} \\
\boldsymbol{x}_{k+1}-\overline{\boldsymbol{x}}_{k+1} \\
\boldsymbol{n}_{k+1}-\overline{\boldsymbol{n}}_{k+1}
\end{array}\right]=\left[\begin{array}{c}
0 \\
0 \\
0 \\
0 \\
\Delta \hat{\boldsymbol{z}}_{n k} \\
\Delta \boldsymbol{y}_{k+1}
\end{array}\right]-\left[\begin{array}{c}
\boldsymbol{v}_{\tilde{w} k} \\
\boldsymbol{v}_{P k} \\
\boldsymbol{v}_{\phi k} \\
\boldsymbol{v}_{x k} \\
\boldsymbol{v}_{n k} \\
\boldsymbol{v}_{k}
\end{array}\right]
$$

The remaining operations of the measurement update and dynamic propagation are accomplished by performing orthogonal/upper triangular $(\mathrm{QR})$ factorization ${ }^{10}$ of the large block matrix into an orthonormal matrix $T$ and an upper triangular matrix. One then multiplies both sides of Eq. (53) by $T^{\mathrm{T}}$. The large block matrix is made upper triangular and the following square-root information equations emerge.

$$
\left[\begin{array}{ccccc}
\hat{R}_{\tilde{w} \tilde{w} k} & \hat{R}_{\tilde{w} P k+1} & \hat{R}_{\tilde{w} \phi k+1} & \hat{R}_{\tilde{w} x k+1} & \hat{R}_{\tilde{w} n k+1} \\
0 & \hat{R}_{P P k+1} & \hat{R}_{P \phi k+1} & \hat{R}_{P x k+1} & \hat{R}_{P n k+1} \\
0 & 0 & \hat{R}_{\phi \phi k+1} & \hat{R}_{\phi x k+1} & \hat{R}_{\phi n k+1} \\
0 & 0 & 0 & \hat{R}_{x x k+1} & \hat{R}_{x n k+1} \\
0 & 0 & 0 & 0 & \hat{R}_{n n k+1} \\
0 & 0 & 0 & 0 & 0
\end{array}\right]\left[\begin{array}{c}
\tilde{\boldsymbol{w}}_{k} \\
\Delta \boldsymbol{b}_{P k+1} \\
\Delta \boldsymbol{b}_{\phi k+1} \\
\Delta \boldsymbol{x}_{k+1} \\
\Delta \boldsymbol{n}_{k+1}
\end{array}\right]=\left[\begin{array}{c}
\Delta \boldsymbol{z}_{\tilde{w} k} \\
\Delta \boldsymbol{z}_{P k+1} \\
\Delta \boldsymbol{z}_{\phi k+1} \\
\Delta \boldsymbol{z}_{x k+1} \\
\Delta \boldsymbol{z}_{n k+1} \\
\Delta \boldsymbol{z}_{r e s}
\end{array}\right]-\left[\begin{array}{c}
\hat{\boldsymbol{v}}_{\tilde{w} k+1} \\
\hat{\boldsymbol{v}}_{P k+1} \\
\hat{\boldsymbol{v}}_{\phi k+1} \\
\hat{\boldsymbol{v}}_{x k+1} \\
\hat{\boldsymbol{v}}_{n k+1} \\
\boldsymbol{v}_{r e s}
\end{array}\right],
$$

where the matrices along the diagonal of the large block matrix are all square, upper-triangular matrices of appropriate dimension, and the matrices above the diagonal of the large block matrix are fully populated and appropriately dimensioned. In Eq. (54), the quantities in the vector that multiplies the large block matrix on the left-hand side have been renamed for brevity to be $\Delta \boldsymbol{b}_{P k+1}=\boldsymbol{b}_{P k+1}-\overline{\boldsymbol{b}}_{P k+1}, \Delta \boldsymbol{b}_{\phi k+1}=\boldsymbol{b}_{\phi k+1}-\overline{\boldsymbol{b}}_{\phi k+1}$, $\Delta \boldsymbol{x}_{k+1}=\boldsymbol{x}_{k+1}-\overline{\boldsymbol{x}}_{k+1}$, and $\Delta \boldsymbol{n}_{k+1}=\boldsymbol{n}_{k+1}-\overline{\boldsymbol{n}}_{k+1}$. The nonhomogeneous vector on the right-hand side of Eq. (54), the one involving $\Delta \boldsymbol{z}_{\tilde{w} k}, \Delta \boldsymbol{z}_{P k+1}$, etc., equals the nonhomogeneous vector from Eq. (53), the one involving $\Delta \hat{\boldsymbol{z}}_{n k}$ and $\Delta \boldsymbol{y}_{k+1}$, left-multiplied by $T^{\mathrm{T}}$. The residual error vector, $\Delta \boldsymbol{z}_{\text {res }}$, is the SRIF equivalent of a Kalman filter innovation. The noise vector has been transformed in the same way. It remains a zero-mean, identity-covariance white noise vector. 
With the equations in this form, one can compute the optimal estimate of the integer vector $\Delta \boldsymbol{n}_{k+1}$ before computing the estimates of the real-valued vectors $\Delta \boldsymbol{b}_{P k+1}, \Delta \boldsymbol{b}_{\phi k+1}$, and $\Delta \boldsymbol{x}_{k+1}$. The optimal estimate of $\Delta \boldsymbol{n}_{k+1}$ is found by solving the following integer linear least squares (ILLS) problem.

$$
\begin{array}{ll}
\text { find: } & \Delta \boldsymbol{n}_{k+1} \\
\text { to minimize: } & J=\frac{1}{2}\left[\hat{R}_{n n k+1} \Delta \boldsymbol{n}_{k+1}-\Delta \boldsymbol{z}_{n k+1}\right]^{\mathrm{T}}\left[\hat{R}_{n n k+1} \Delta \boldsymbol{n}_{k+1}-\Delta \boldsymbol{z}_{n k+1}\right] \\
\text { subject to: } & \boldsymbol{\Delta} \boldsymbol{n}_{k+1} \text { is an integer-valued vector }
\end{array}
$$

For details on how to solve such ILLS problems, see Refs. 11 and 12. Let $\Delta \hat{\boldsymbol{n}}_{k+1}$ be the value of $\Delta \boldsymbol{n}_{k+1}$ that minimizes Eq. (55). The next step is to compute $\Delta \hat{\boldsymbol{z}}_{n k+1}$ based on $\Delta \boldsymbol{z}_{n k+1}$ and $\Delta \hat{\boldsymbol{n}}_{k+1}$. The quantity $\Delta \hat{\boldsymbol{z}}_{n k+1}$ will be used in the next recursion of the filter, when $k$ is incremented by one.

$$
\Delta \hat{\boldsymbol{z}}_{n k+1}=\Delta \boldsymbol{z}_{n k+1}-\hat{R}_{n n k+1} \Delta \hat{\boldsymbol{n}}_{k+1}
$$

The optimal a posteriori estimates of $\Delta \boldsymbol{b}_{P k+1}, \Delta \boldsymbol{b}_{\phi k+1}$, and $\Delta \boldsymbol{x}_{k+1}$ are found by back substitution.

$$
\begin{gathered}
\Delta \hat{\boldsymbol{x}}_{k+1}=\hat{R}_{x x k+1}^{-1}\left[\Delta \boldsymbol{z}_{x k+1}-\hat{R}_{x n k+1} \Delta \hat{\boldsymbol{n}}_{k+1}\right] \\
\Delta \hat{\boldsymbol{b}}_{\phi k+1}=\hat{R}_{\phi \phi k+1}^{-1}\left[\Delta \boldsymbol{z}_{\phi k+1}-\hat{R}_{\phi x k+1} \Delta \hat{\boldsymbol{x}}_{k+1}-\hat{R}_{\phi n k+1} \Delta \hat{\boldsymbol{n}}_{k+1}\right] \\
\Delta \hat{\boldsymbol{b}}_{P k+1}=\hat{R}_{P P k+1}^{-1}\left[\Delta \boldsymbol{z}_{P k+1}-\hat{R}_{P \phi k+1} \Delta \hat{\boldsymbol{b}}_{\phi k+1}-\hat{R}_{P x k+1} \Delta \hat{\boldsymbol{x}}_{k+1}-\hat{R}_{P n k+1} \Delta \hat{\boldsymbol{n}}_{k+1}\right]
\end{gathered}
$$

The a posteriori estimates of $\hat{\boldsymbol{b}}_{P k+1}, \hat{\boldsymbol{b}}_{\phi k+1}, \hat{\boldsymbol{x}}_{k+1}$, and $\hat{\boldsymbol{n}}_{k+1}$ can be computed by adding the a posteriori estimates of the perturbation to the a priori estimates.

$$
\begin{gathered}
\hat{\boldsymbol{b}}_{P k+1}=\overline{\boldsymbol{b}}_{P k+1}+\Delta \hat{\boldsymbol{b}}_{P k+1} \\
\hat{\boldsymbol{b}}_{\phi k+1}=\overline{\boldsymbol{b}}_{\phi k+1}+\Delta \hat{\boldsymbol{b}}_{\phi k+1} \\
\hat{\boldsymbol{x}}_{k+1}=\overline{\boldsymbol{x}}_{k+1}+\Delta \hat{\boldsymbol{x}}_{k+1} \\
\hat{\boldsymbol{n}}_{k+1}=\overline{\boldsymbol{n}}_{k+1}+\Delta \hat{\boldsymbol{n}}_{k+1}
\end{gathered}
$$

At this point, filtering over the time step from $t_{k}$ to $t_{k+1}$ is complete, and the a posteriori quantities may be passed forward to initialize the next recursion of the filter. Section IV.C lists those matrices and vectors that must be passed forward from one time step to the next and gives a synopsis of the algorithm.

\section{B. Deletion of Inactive Pseudorange and Carrier Phase Biases and Doppler Shift Ambiguities}

Over time, as the spacecraft accumulates passes over the various ground stations, the dimensions of the $\boldsymbol{b}_{P}$ and $\boldsymbol{b}_{\phi}$ vectors grow. At the completion of a pass over a ground station, the biases associated with that pass will no longer enter any measurement equations and will have no further effect on the state estimate. It is efficient to drop those elements out of $\boldsymbol{b}_{P}$ and $\boldsymbol{b}_{\phi}$ in order to limit their size. Consider the combined vector of biases $\boldsymbol{b}=\left[\boldsymbol{b}_{P}^{\mathrm{T}} \boldsymbol{b}_{\phi}^{\mathrm{T}}\right]^{\mathrm{T}}$ at time $t_{k+1}$, when there are $N_{b}$ biases in the vector,

$$
\boldsymbol{b}_{k+1}=\left[\begin{array}{llllll}
b_{1} & b_{2} & \ldots & b_{i} & \ldots & b_{N_{b}}
\end{array}\right]^{\mathrm{T}}
$$

Note that in the remainder of this section, the two bias vectors $\boldsymbol{b}_{P}$ and $\boldsymbol{b}_{\phi}$ are concatenated together to form a single, simpler-to-write bias vector $\boldsymbol{b}$. The notation for all other quantities has been adjusted accordingly by forming larger block matrices or concatenations of vectors.

Suppose that the ground-station pass associated with bias $b_{i}$ in $\boldsymbol{b}$ is complete. Therefore, $b_{i}$ will no longer appear in any measurement equations, and it can be discarded. This can be accomplished in three 
steps. First, one permutes the elements of the composite vector on the left-hand side of Eq. 54 such that the element associated with $b_{i}$ is the first element of the composite vector,

$$
\left[\begin{array}{lll}
\Delta b_{i} & \tilde{\boldsymbol{w}}_{k}^{\mathrm{T}} \Delta \tilde{\boldsymbol{b}}_{k+1}^{\mathrm{T}} \Delta \boldsymbol{x}_{k+1}^{\mathrm{T}} \Delta \boldsymbol{n}_{k+1}^{\mathrm{T}}
\end{array}\right]^{\mathrm{T}}
$$

where $\Delta \tilde{\boldsymbol{b}}_{k+1}$ is $\Delta \boldsymbol{b}_{k+1}$ with $\Delta b_{i}$ excised. This permutation necessitates a corresponding permutation of the columns of $\hat{R}_{b b k+1}$ and $\hat{R}_{\tilde{w} b k+1}$ in the left-hand side of Eq. (54), which permutation destroys the upper-triangularity of the block matrix.

Second, in order to restore the upper-triangularity of the permuted large block matrix, a new QR factorization must be applied to Eq. (54). The resulting equation is

$$
\left[\begin{array}{ccccc}
\hat{R}_{b_{i} b_{i}} & \hat{R}_{b_{i} \tilde{w} k} & \hat{R}_{b_{i} b k+1} & \hat{R}_{b_{i} x k+1} & \hat{R}_{b_{i} n k+1} \\
0 & \hat{R}_{\tilde{w} \tilde{w} k} & \hat{R}_{\tilde{w} \tilde{b} k+1} & \hat{R}_{\tilde{w} x k+1} & \hat{R}_{\tilde{w} n k+1} \\
0 & 0 & \hat{R}_{\tilde{b} \tilde{b} k+1} & \hat{R}_{\tilde{b} x k+1} & \hat{R}_{\tilde{b} n k+1} \\
0 & 0 & 0 & \hat{R}_{x x k+1} & \hat{R}_{x n k+1} \\
0 & 0 & 0 & 0 & \hat{R}_{n n k+1} \\
0 & 0 & 0 & 0 & 0
\end{array}\right]\left[\begin{array}{c}
\Delta \boldsymbol{b}_{i} \\
\tilde{\boldsymbol{w}}_{k} \\
\Delta \tilde{\boldsymbol{b}}_{k+1} \\
\Delta \boldsymbol{x}_{k+1} \\
\Delta \boldsymbol{n}_{k+1}
\end{array}\right]=\left[\begin{array}{c}
\Delta \boldsymbol{z}_{b_{i}} \\
\Delta \boldsymbol{z}_{\tilde{w} k} \\
\Delta \boldsymbol{z}_{\tilde{b} k+1} \\
\Delta \boldsymbol{z}_{x k+1} \\
\Delta \boldsymbol{z}_{n k+1} \\
\Delta \boldsymbol{z}_{r e s}
\end{array}\right]-\left[\begin{array}{c}
\hat{\boldsymbol{v}}_{b_{i}} \\
\hat{\boldsymbol{v}}_{\tilde{w} k+1} \\
\hat{\boldsymbol{v}}_{b k+1} \\
\hat{\boldsymbol{v}}_{x k+1} \\
\hat{\boldsymbol{v}}_{n k+1} \\
\boldsymbol{v}_{r e s}
\end{array}\right]
$$

Third and finally, the first row of Eq. (66) is dropped from the problem along with the left-most column of the large block matrix on the left-hand side of Eq (66).

One might be tempted to drop integer-valued Doppler shift ambiguities in an analogous manner, if they too apply to completed ground-station passes. In fact, this produces a suboptimal solution. If the given integer element of $\boldsymbol{n}$ is known with a very high degree of certainty when it is dropped, then optimality can be maintained, but with a modified dropping procedure. ${ }^{2}$ In general, it is difficult to be certain that the estimated integers are exactly correct. By carrying the integers forward forever, the optimal solution is found. A problem with this approach, however, is that the integer vector grows in length. The resulting ILLS problem can become very costly to solve after each measurement update. A reasonable suboptimal solution is found by dropping an integer-valued state after a significant interval has elapsed since it last entered a measurement equation. The optimal and suboptimal integer dropping methods are treated in more detail in Ref. 2. In the present work, the integers are dropped as if they were real-valued after a reasonable time has elapsed since they last entered a measurement equation.

\section{Algorithm}

The following steps summarize the algorithm presented in this paper.

1. Set $k=0$

2. Numerically integrate from $t_{k}$ to $t_{k+1}$ to find $\overline{\boldsymbol{x}}_{k+1}, \Phi_{k}$, and $\Gamma_{k}$.

3. Set up Eq. (53) with the known quantities from the previous time step, or with the initial estimates if $k=0$, and append the measurements accumulated between time $t_{k}$ and time $t_{k+1}$. The quantities carried forward from the last time step are $\hat{R}_{P P k}, \hat{R}_{P \phi k}, \hat{R}_{P x k}, \hat{R}_{P n k}, \hat{R}_{\phi \phi k}, \hat{R}_{\phi x k}, \hat{R}_{\phi n k}, \hat{R}_{x x k}, \hat{R}_{x n k}$, $\hat{R}_{n n k}, \Delta \hat{z}_{n k}$, and the a posteriori bias, state, and ambiguity estimates from the previous time step.

4. QR factorize and transform to get Eq. (54).

5. Drop biases that will no longer appear in any measurement equations, as per Section IV.B.

6. If dropping integers, drop those which last appeared in a measurement equation more than $N_{s}$ time steps ago, where $N_{s}$ is some threshold value, as per Ref. 2.

7. Solve the ILLS problem for $\Delta \hat{\boldsymbol{n}}_{k+1}$.

8. Solve for $\Delta \hat{\boldsymbol{z}}_{n k+1}, \Delta \hat{\boldsymbol{x}}_{k+1}, \Delta \hat{\boldsymbol{b}}_{\phi k+1}$, and $\Delta \hat{\boldsymbol{b}}_{P k+1}$ using Eqs. (56)-(59).

9. Solve for the estimates $\hat{\boldsymbol{b}}_{P k+1}, \hat{\boldsymbol{b}}_{\phi k+1}, \hat{\boldsymbol{x}}_{k+1}$, and $\hat{\boldsymbol{n}}_{k+1}$ using Eqs. (60)-(63)

10. Set $k=k+1$, and go to Step 2 . 


\section{Truth Model Simulation}

In order to evaluate the effectiveness of the filter, it was applied to data that were derived from a truth model simulation. The truth model simulation generates truth values for the process noise polynomial coefficients, and uses them to generate the truth state vector time history. The simulation synthesizes measurements by using the true state history in the measurement equation, and by adding in the effects of phase bias, pseudorange bias, Doppler shift ambiguity, and measurement noise. The filter is then able to operate on these synthesized measurements, and the results can be compared to the true values from the simulation.

The values of the process noise polynomial coefficients were generated using a pseudo-random number generator to draw samples from normal distributions with statistics as described in Section II.F. In the simulation, fourth-order Chebyshev polynomials were used to approximate process noise. The optimal values of the " $\alpha$ " coefficients of Eq. (24) for fourth-order process noise are taken from Ref. 1 . Once the process noise polynomial coefficients for an interval are chosen, the process noise affecting the system can be found at any point on the interval by evaluating the weighted sum of polynomials, Eq.( 14).

Given realistic initial conditions for a LEO satellite and the process noise polynomial coefficients for all integration intervals, the truth model simulation numerically integrates the dynamics equations forward in time in order to generate the truth orbit. The truth model simulation uses the same dynamics equations as the filter.

The truth model simulation starts with the reception times of the measurements at all ground stations as givens, and it uses the constraint equation and the state interpolation from the dense-output numerical integration to calculate the time of transmission from the satellite and the location of the satellite at that time. From this information, the time history of received beat carrier phase is generated. The pseudorange bias is generated by adding white, Gaussian, zero-mean noise with standard deviation of 1 microsecond to the arrival times. A random, constant offset added to the phase history for each pass over each ground station accounts for the effects of phase bias. The Doppler shift integer ambiguity, $n_{i}$, is generated by sampling the set $\{-3,-2,-1,0,1,2,3\}$ with equal probability and using this value as the number of cycles that ramps linearly with measurement number, as per Eq. (26). The random component of the carrier phase measurement error, $v$ in Eq. (26), is sampled from a zero-mean Gaussian distribution with standard deviation of $4.6 \mathrm{~mm}$.

The truth model simulation used for the purposes of this paper included five simulated ground stations located in the continental United States. Figure 3 shows the locations of the five ground stations. The orbits used in the truth model simulation were nearlycircular, nearly-polar orbits with period of about 100 minutes. Each ground station recorded measurements at a rate of $1.1 \mathrm{~Hz}$ when the satellite was above 10 degrees elevation with respect to the ground station. The satellite's clock model parameters were chosen to give it characteristics approximating those of an ovenized crystal oscillator: $h_{0}=$ $2 \mathrm{E}-22 \mathrm{~s}$ and $h_{-2}=6.1 \mathrm{E}-221 / \mathrm{s}$, which gives a minimum root Allen variance of $3.56 \mathrm{E}-11$ at a time delay of $0.158 \mathrm{~s}$.

The truth model simulation tests

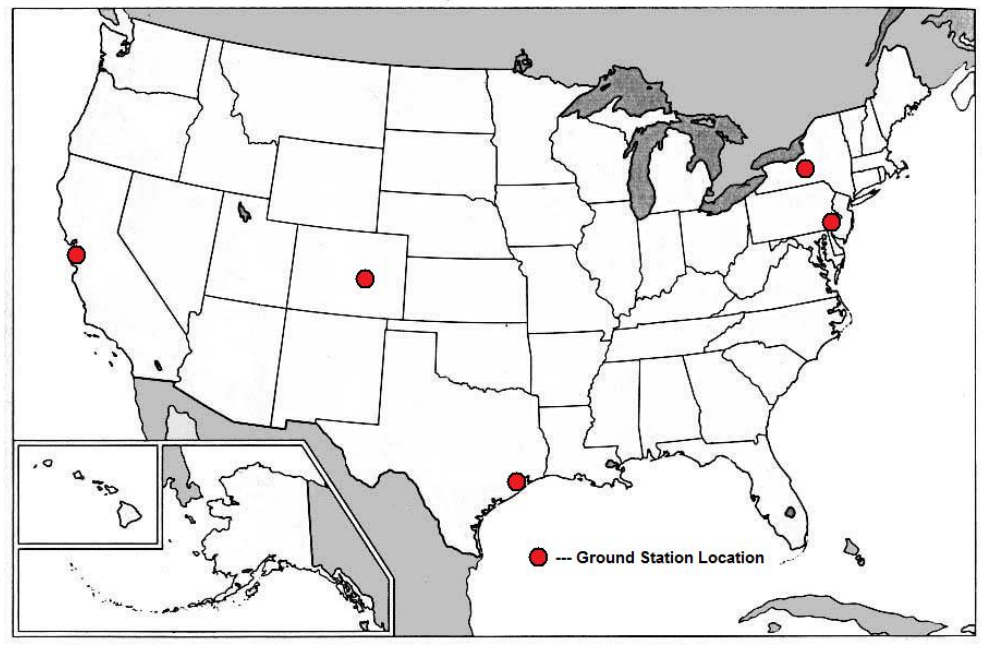

Figure 3. The approximate locations of the five simulated ground stations.

provided a means to evaluate filter performance under controlled conditions with known truth states. The availability of truth states made it easy to investigate filter convergence by perturbing the initial state estimate around the true initial state. Using the truth states, it was also possible to carry out statistical consistency checks of the filter that would otherwise have been impossible to perform. The truth model also provided a means of quickly evaluating filter performance under ideal conditions. 


\section{Results}

Several simulations were run, all with the same initial conditions, but each with new, randomly selected process noise. Filter convergence was investigated using some of the shorter simulations by running the filter several times with different initial estimation errors and covariances. For each of the filter runs, filter consistency and performance were checked. Filter performance results given below are averaged over five independent 25-hour filter runs. This duration yields three distinct sets of passes over the continental United States. Also discussed in detail are results from a single, characteristic 25-hour run. No attempt was made to investigate different types of orbits. The truth model simulation was used only to ensure that the filter produces reasonable results.

The filter reliably converges to reasonable orbit estimates - i.e., position estimate errors of less than one meter - in only one pass over the ground stations if the initial position error is less than about $25 \mathrm{~m}$ and the initial velocity error is less than about $1 \mathrm{~m} / \mathrm{s}$. The filter converges in two to three orbits when the initial position error is less than a few hundred meters and the initial velocity error is less than a couple meters per second. The filter is able to recover from initial errors of larger magnitude, e.g. initial position errors of over $1 \mathrm{~km}$, but the time to converge increases to several orbits, and the spacecraft clock offset estimation errors are larger.

Note that a linear combination of the clock error, $\delta t$, and the carrier phase and pseudorange biases, $b_{1}$, $b_{2}, b_{3}$, etc. is unobservable. This does not affect the observability of the orbit or the clock frequency states, because $\delta t, b_{1}, b_{2}, b_{3}$, etc. are merely "nuisance" parameters in the present problem. A possible future upgrade might include true pseudorange measurements at the ground stations, as opposed to the biased pseudorange measurements currently in use. Such an upgrade would make $\delta t$, and the biases $b_{1}, b_{2}, b_{3}$, etc. fully observable, but would require that the satellite tag each transmission with its own clock time at time of transmission.

Filter consistency was evaluated in order to ensure filter optimality. In the present work, normalized estimation error squared (NEES) and normalized innovation squared (NIS) were considered, as these two consistency tests indicate whether or not the state estimation errors and the measurement prediction errors are zero-mean with covariance as predicted by the filter. ${ }^{13}$

NEES is usually defined as

$$
\epsilon(k)=\left[\hat{\boldsymbol{x}}_{k}-\boldsymbol{x}_{k}\right]^{\mathrm{T}} P_{k}^{-1}\left[\hat{\boldsymbol{x}}_{k}-\boldsymbol{x}_{k}\right],
$$

where $P_{k}$ is the a posteriori covariance estimate which normalizes the state estimation errors. However, the form of the state vector of the present filter makes it necessary to redefine the NEES as

$$
\epsilon(k)=\left[\begin{array}{c}
\hat{\boldsymbol{x}}_{k}-\boldsymbol{x}\left(t_{k}\right) \\
\hat{\boldsymbol{n}}_{k}-\boldsymbol{n}\left(t_{k}\right)
\end{array}\right]^{\mathrm{T}}\left[\begin{array}{ll}
\hat{R}_{x x k} & \hat{R}_{x n k}
\end{array}\right]^{\mathrm{T}}\left[\begin{array}{ll}
\hat{R}_{x x k} & \hat{R}_{x n k}
\end{array}\right]\left[\begin{array}{c}
\hat{\boldsymbol{x}}_{k}-\boldsymbol{x}\left(t_{k}\right) \\
\hat{\boldsymbol{n}}_{k}-\boldsymbol{n}\left(t_{k}\right)
\end{array}\right] .
$$

In cases where the Doppler shift ambiguities are estimated exactly correctly, this form of the NEES is equivalent to the standard form given in Eq. 67. If the filter is consistent, each $\epsilon(k)$ should be drawn from a $\chi^{2}$ distribution with $n_{x}$ degrees of freedom, where $n_{x}$ is the number of elements in the state vector, $\boldsymbol{x}$. If $\epsilon(k)$ comes from such a distribution, then bounds $r_{1}$ and $r_{2}$ can be computed such that $\epsilon(k)$ should fall in the interval $\left[r_{1}, r_{2}\right]$ with some chosen probability. Fig. 4 shows the NEES for a single run of the filter along with the $90 \%$ probability region plotted on a logarithmic scale. For this particular run, the NEES fell inside the $90 \%$ probability region $97.7 \%$ of the time. Over all runs, the NEES fell inside the $90 \%$ probability region between $87.2 \%$ and $97.7 \%$ of the time, with an average of $90.5 \%$ of NEES inside the $90 \%$ probability region.

The other consistency test that was run is the normalized innovation squared. The NIS is

$$
\epsilon_{\nu}(k)=\Delta z_{r e s}(k)^{\mathrm{T}} \Delta z_{\text {res }}(k),
$$

The quantity $\epsilon_{\nu}(k)$ should be drawn from a $\chi^{2}$ distribution with $n_{z}(k)$ degrees of freedom, where $n_{z}(k)$ is the number of measurements that arrive between time $t_{k}$ and time $t_{k+1}$. Note that were it not for the presence of integer states, $\epsilon_{\nu}(k)$ would equal the usual NIS, $\nu(k)^{\mathrm{T}} S(k)^{-1} \nu(k)$, where $\nu(k)$ is the usual Kalman filter innovation and $S(k)$ is its covariance.

As was the case for the NEES, an interval can be defined, based on the properties of the $\chi^{2}$ distribution, in which $\epsilon_{\nu}(k)$ should fall with some probability, if the filter is consistent. Fig. 5 shows an example of the NIS during a single pass over the United States along with the $90 \%$ probability region plotted on a logarithmic scale. Note that the bounds of the $90 \%$ probability region vary from one time step to the next, because 


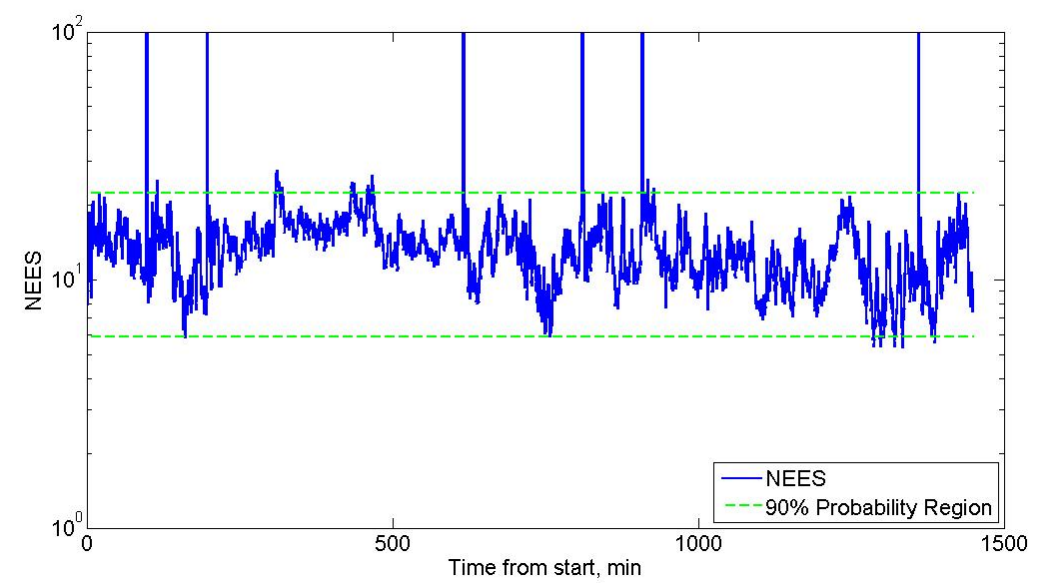

Figure 4. Normalized estimation error squared for a single run of the filter along with the $90 \%$ probability region.

$n_{z}(k)$ changes as ground stations come into view or drop over the horizon. For periods during which there are no measurements, $n_{z}(k)$ is zero and $\epsilon_{\nu}(k)$ is undefined. For this particular run, where $\epsilon_{\nu}(k)$ is defined, it falls within the $90 \%$ probability region $88.5 \%$ of the time. Over all runs, NIS fell inside the $90 \%$ probability region between $87.5 \%$ and $89.2 \%$ of the time, with an average of $88.4 \%$ of NIS inside the $90 \%$ probability region.

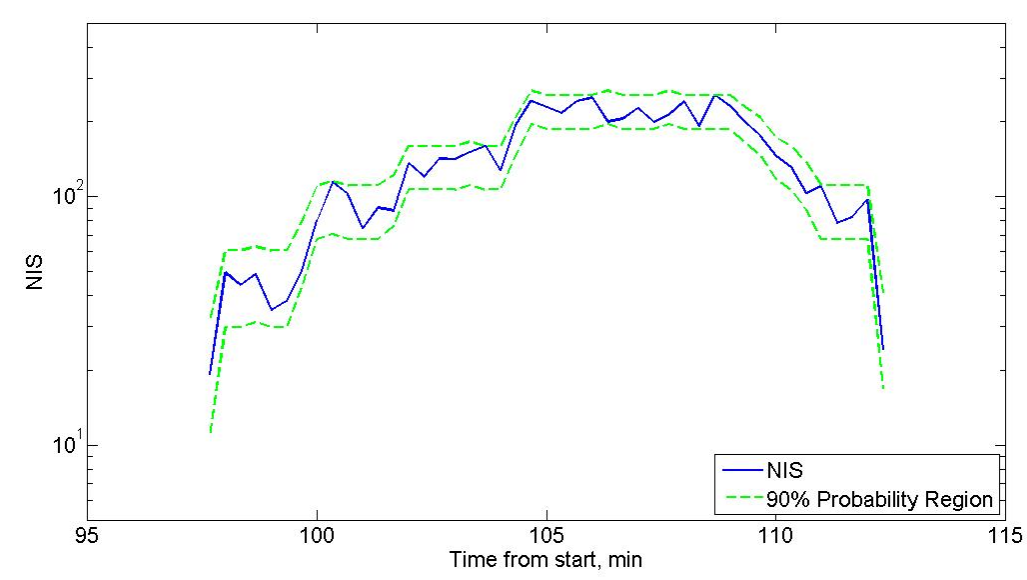

Figure 5. Normalized innovation squared for a single pass over the ground stations along with the $90 \%$ probability region.

The results of the consistency checks indicate that the filter's state estimate errors and measurement prediction errors are zero-mean and that they both have covariance as predicted by the filter.

The position and velocity accuracy of the filter depend strongly on time. During times of ground station contact, the accuracy is best. Between ground station contacts, the level of error standard deviation drifts upward. Figures 6 and 7 illustrate this fact. They show typical time histories of position estimation error magnitude and velocity estimation error magnitude over a 25-hour period. Both figures use logarithmic vertical scales for their respective errors and linear horizontal time scales. The sharp reductions in error magnitude correspond to times at which one or more ground stations come into view. While the filter is processing measurements, the error in the position estimate settles to sub-centimeter levels, and the error in the velocity estimate typically falls below $0.1 \mathrm{~mm} / \mathrm{s}$. During a coasting period of a single orbit, the position estimation error magnitude grows to about the 10-meter level, and the velocity estimation error magnitude grows to about $1 \mathrm{~cm} / \mathrm{s}$. Longer coasting periods also occur, for instance from about 200 to 600 minutes 
in Fig. 6, which correspond to about four full orbits. During these long coasting periods, across all runs considered, the position estimation error grew to a maximum of $681.3 \mathrm{~m}$ with an average of $305.5 \mathrm{~m}$.

The same data that went into Fig. 6 are broken into their along-track, altitude, and cross-track components and re-plotted in Fig. 8. As expected, the orbit plane is determined very well; the peak cross-track position estimate error in this run, after the initial transient, is only 2.1 meters and 0.10 meters RMS. Also as expected, it is the along-track direction with the highest estimation error-about 681.3 meters following the first long coasting period, but only 201 meters RMS. The maximum and RMS position estimation errors are summarized in Table 1. Note that these errors are all calculated after waiting for the initial transient to die out.

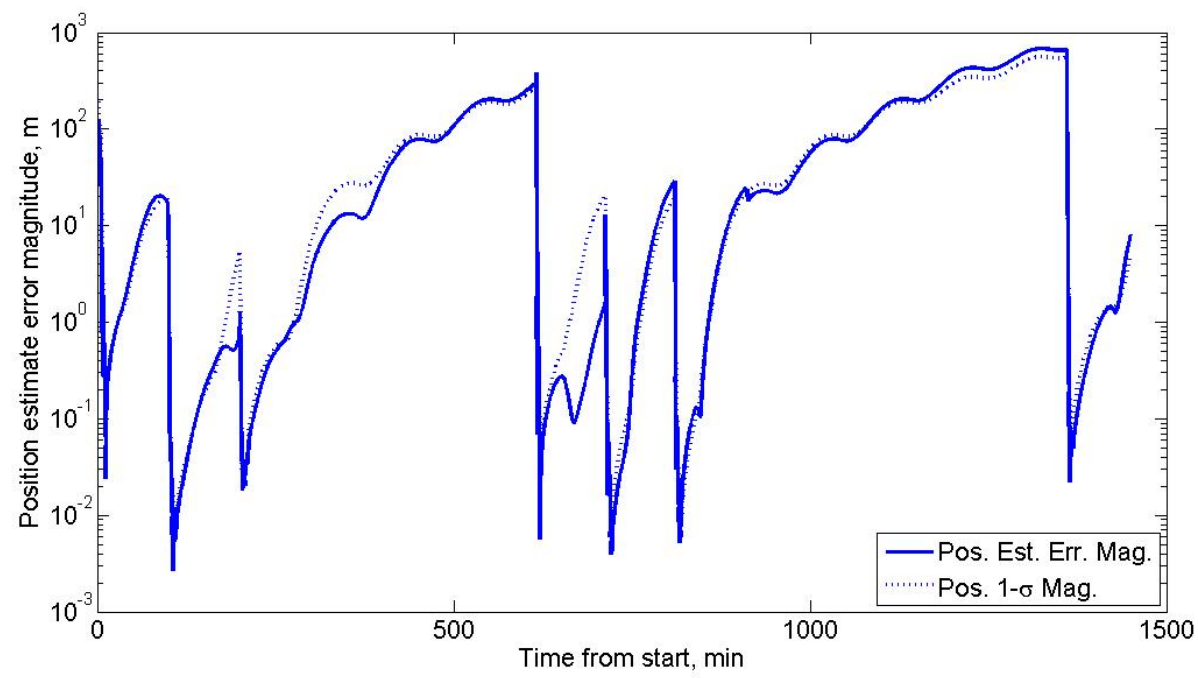

Figure 6. Position error magnitude time history for a typical 25-hour period.

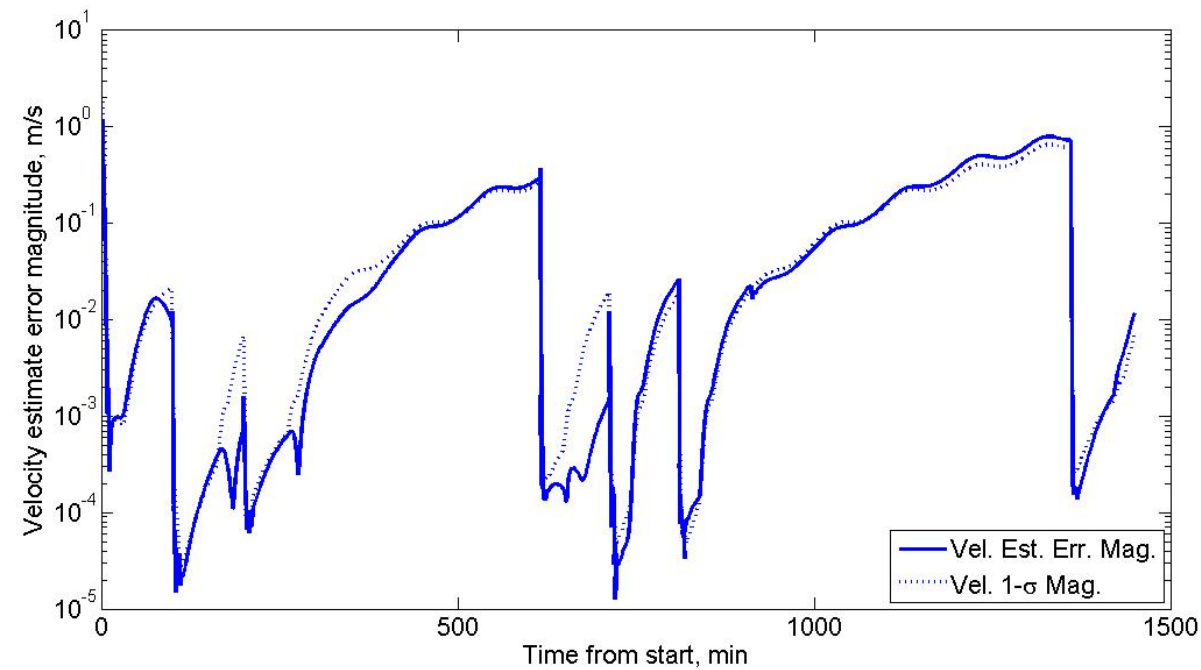

Figure 7. Velocity error magnitude time history for a typical 25-hour period.

Note that the best results here, with position accuracy better than $1 \mathrm{~cm}$ and velocity accuracy better than $0.1 \mathrm{~mm} / \mathrm{s}$ are over optimistic. When real effects are considered that are not presently modeled, the accuracy will degrade. Such effects include residual force model errors, as well as errors in models of the ionospheric and neutral atmosphere delays. 


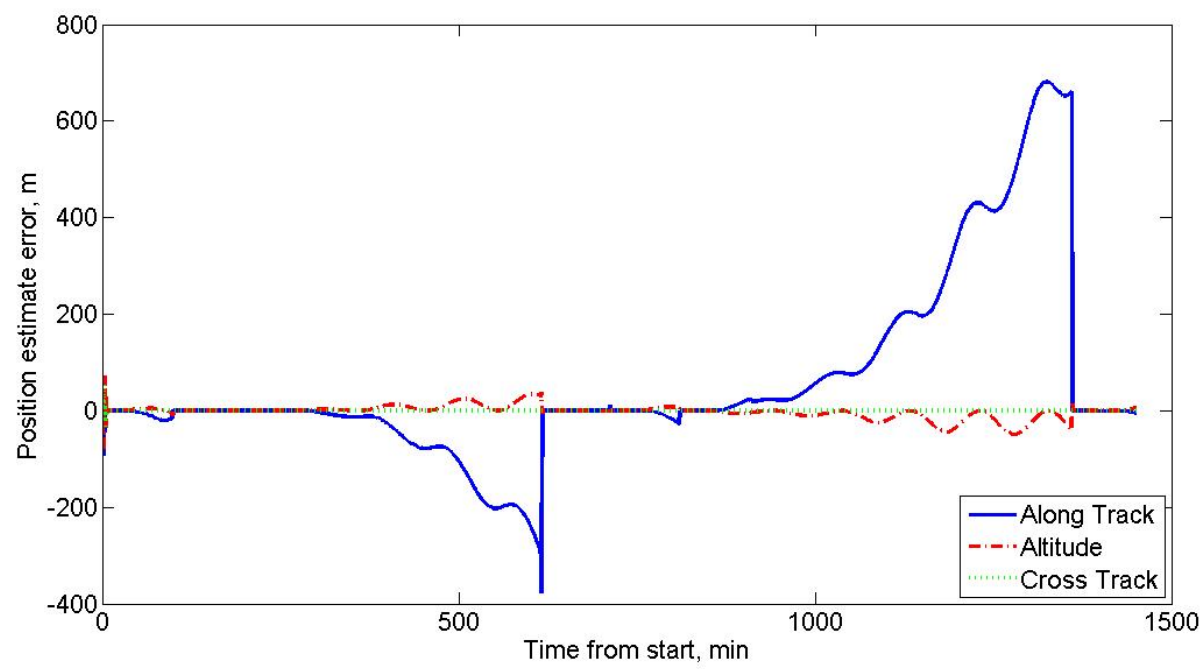

Figure 8. Position error component time histories for a typical 25-hour period.

Table 1. Satellite Position Estimation Error Summary (Truth Model Data)

5-Run Average

Maximum Error (m) RMS Error (m)

Along-Track

Altitude

681.26

49.25

2.51
112.17

8.03

0.08 


\section{Summary and Conclusions}

A new Kalman filtering technique was applied in an orbit determination setting using time-division multiple access (TDMA) radio communication signals that were not originally intended for this application. The orbit determination filter has two significant new features. The first is an ability to deal directly and consistently with the unknown measurement effectiveness times that result from using ground-based radio navigation data. The second new feature is the estimation of integer Doppler shift ambiguities. Such ambiguities tend to arise for TDMA signals. The implicit nature of the measurement effectiveness times was dealt with by using dense-output Runge-Kutta numerical integration throughout to provide interpolated values for the state at all times. This feature also required the use of a piecewise polynomial process noise model. In order to handle the integer-valued Doppler shift ambiguities in a sensible way, the square-root information extended Kalman filter solved an integer linear least squares problem before estimating the real-valued portion of the state vector.

In truth model simulation, the algorithm determines the orbits of low Earth orbit satellites with RMS position error magnitude of only 110 meters overall. The peak position error magnitude observed of about 680 meters occurs after nearly 7 hours without measurements. Statistical consistency checks on the state estimation errors and the residuals showed that the filter functions as expected. If the periods during which no measurements were available were shortened by increasing the number and geographical diversity of ground stations, the peak position estimate errors could be reduced significantly. Longer filtering runs might also reduce this error.

\section{References}

\footnotetext{
${ }^{1}$ Mohiuddin, S. and Psiaki, M. L., "Continuous-Time Kalman Filtering with Implicit Discrete Measurement Times," AIAA Guidance, Navigation, and Control Conference, Washington, D.C., Aug. 2009, pp. 4574-4607.

${ }^{2}$ Psiaki, M. L., "Kalman Filtering and Smoothing to Estimate Real-Valued States and Integer Constants," AIAA Guidance, Navigation, and Control Conference Proceedings, Washington, D.C., Aug. 2009, AIAA Paper Number 2009-5972.

${ }^{3}$ Brown, R. G. and Hwang, P. Y. C., Introduction to Random Signals and Applied Kalman Filtering with MATLAB exercises and solutions, Wiley, New York, 3rd ed., Nov. 1996.

${ }^{4}$ Lundberg, J. and Schutz, B., "Recursion Formulas for Legendre Functions for Use with Nonsingular Geopotential Models," Journal of Guidance, Control, and Dynamics, Vol. 11, No. 1, January-February 1988, pp. 31-38.

${ }^{5}$ Montenbruck, O. and Gill, E., Satellite Orbits: Models, Methods and Applications, Springer, Berlin, Heidelberg, and New York, 2005.

${ }^{6}$ Nørsett, S. P., Wanner, G., and Hairer, E., Solving ordinary differential equations, Springer-Verlag, Berlin, 1987.

${ }^{7}$ Mendes, V., Modeling the Neutral-Atmospheric Propagation Delay in Radiometric Space Techniques, Ph.D. thesis, Geodesy and Geomatics Engineering, University of New Brunswick, April 1999.

${ }^{8}$ Misra, P. and Enge, P., Global Positioning System: Signals, Measurements, and Performance, Ganga-Jamuna Press, Lincoln, Massachusetts, 2nd ed., 2006.

${ }^{9}$ Bierman, G. J., Factorization Methods for Discrete Sequential Estimation, Academic Press, New York, New York, 1977, pp 69-76, 115-122, 214-217.

${ }^{10}$ Gill, P. E., Murray, W., and Wright, M. H., Practical Optimization, Academic Press, San Diego, California, 1981, pp $37-40$.

${ }^{11}$ Teunissen, P. J. G., "The Least-Squares Ambiguity Decorrelation Adjustment: A Method for Fast GPS Integer Ambiguity Estimation," Journal of Geodesy, Vol. 70, No. 1-2, Nov. 1995, pp. 65-82.

${ }^{12}$ Psiaki, M. and Mohiuddin, S., "Global Positioning System Integer Ambiguity Resolution Using Factorized Least Squares Techniques," Journal of Guidance, Control, and Dynamics, Vol. 30, No. 2, Mar-Apr 2007, pp. 346-356.

${ }^{13}$ Bar-Shalom, Y., Li, X. R., and Kirubarajan, T., Estimation with Applications to Tracking and Navigation, John Wiley and Sons, New York, New York, 2001, pp 232-236.
} 